University of Nebraska - Lincoln

DigitalCommons@University of Nebraska - Lincoln

Anthony F. Starace Publications

Research Papers in Physics and Astronomy

August 1978

\title{
Graphical approach to the spin-orbit interaction
}

\author{
Keh-Ning Huang \\ University of Nebraska - Lincoln \\ Anthony F. Starace \\ University of Nebraska-Lincoln, astarace1@unl.edu
}

Follow this and additional works at: https://digitalcommons.unl.edu/physicsstarace

Part of the Physics Commons

Huang , Keh-Ning and Starace, Anthony F., "Graphical approach to the spin-orbit interaction" (1978). Anthony F. Starace Publications. 9.

https://digitalcommons.unl.edu/physicsstarace/ 9

This Article is brought to you for free and open access by the Research Papers in Physics and Astronomy at DigitalCommons@University of Nebraska - Lincoln. It has been accepted for inclusion in Anthony F. Starace Publications by an authorized administrator of DigitalCommons@University of Nebraska - Lincoln. 


\title{
Graphical approach to the spin-orbit interaction*
}

\author{
Keh-Ning Huang and Anthony F. Starace ${ }^{\dagger}$ \\ Behlen Laboratory of Physics, The University of Nebraska, Lincoln, Nebraska 68588
}

(Received 28 February 1977)

\begin{abstract}
We derive a new form for the matrix element of the two-particle mutual spin-orbit interaction in the BreitPauli approximation. The complexity of standard tensor-operator expansion techniques is obviated by suitably decomposing the mutual spin-orbit interaction into products of spin and orbital angular-momentum operators, whose matrix elements in the $l m_{l} s m_{s}$ scheme are obtained in a straightforward manner. Graphical representations are given for both the one- and two-particle spin-orbit interactions, thereby permitting the analytical evaluation by graphical techniques of the spin-orbit interaction between arbitrary configurations in any coupling scheme. The graphical procedure for evaluating these matrix elements is demonstrated for $L S$ coupled configurations having two nonequivalent electrons outside closed shells. Various properties of the twoparticle mutual spin-orbit operator are easily illustrated using its graphical representation.
\end{abstract}

\section{INTRODUCTION}

The spin-orbit interaction plays an essential role in the calculation of atomic structure, ${ }^{1-3}$ atomic oscillator strengths, ${ }^{4}$ and other atomic processes. ${ }^{5}$ However, the calculation of spinorbit interaction in many-electron atoms is complicated by the presence of the two-particle mutual spin-orbit interaction. In the older accounts, ${ }^{1}$ the total spin-orbit interaction of a many-electron atom is represented approximately by a sum of one-particle spin-orbit operators of the following form:

$$
V_{s o}=\sum_{i=1}^{N} \zeta\left(r_{i}\right) \overrightarrow{\mathrm{L}}_{i} \cdot \overrightarrow{\mathrm{s}}_{i},
$$

where $N$ is the number of electrons, and $\overrightarrow{\mathrm{L}}_{i}$ and $\overrightarrow{\mathrm{s}}_{i}$ are the orbital and spin angular-momentum operators of the $i$ th electron. The spin-orbit parameter $\zeta\left(r_{i}\right)$ is commonly taken in atomic units as

$$
\zeta\left(r_{i}\right)=\frac{\alpha^{2}}{2} \frac{1}{r_{i}} \frac{\partial V\left(r_{i}\right)}{\partial r_{i}},
$$

where $\alpha$ is the fine-structure constant, and $V\left(r_{i}\right)$ is the effective central potential in which the $i$ th electron moves. Unlike the one-electron atom case, in the many-electron atom the mutual spinorbit interaction cannot in general be interpreted by considering an electron moving in a central field. That is, it cannot be reduced to an effective one-particle spin-orbit operator but must be treated as a two-particle operator.

The mutual spin-orbit interaction between electrons in a many-electron atom is obtained by a series of successive approximations. The relativistic theory for a single electron is described exactly by the Dirac equation, ${ }^{6}$ provided one neglects quantum electrodynamic and field-theoretic effects. The existence of the electron spin gives rise to the one-particle spin-orbit interaction in the single-electron case. For many-electron atoms, the quantum electrodynamic interaction between electrons to the lowest order in $\alpha$ is due to the exchange of a transverse photon. ${ }^{7}$ This lowest-order interaction may be approximated, to terms of order $(v / c)^{2}$, by the Breit interaction. ${ }^{8}$ In the Pauli approximation, ${ }^{9}$ the Breit interaction gives rise to the mutual orbit-orbit, spin-orbit, and spin-spin interactions as well as others. ${ }^{10}$ Although in a complete treatment of multiplet structure all these interactions have to be considered, the spin-orbit interaction is by far the largest one, after the Coulomb interaction, for medium and heavy atoms.

Many studies of the mutual spin-orbit interaction have been carried out. ${ }^{11-23}$ A number of these researches were aimed at deriving a more realistic spin-orbit parameter using Hartree-Fock wave functions. In particular, Blume and Watson ${ }^{17}$ have developed a theory of the spin-orbit parameter for many-electron atoms and applied it to calculations for a number of atoms and ions. Although they obtained a tensor-operator expansion for the mutual spin-orbit interaction, calculations were carried out only within the same configuration for atoms having a single open shell. Using Blume and Watson's tensorial expression for the mutual spin-orbit interaction, Jucys and co-workers $^{21,22}$ obtained matrix elements of the mutual spin-orbit interaction within the same configuration. Also using Blume and Watson's tensorial expression, Jones ${ }^{23}$ calculated its general matrix element in the $l m_{l} s m_{s}$ scheme (which will be referred to as "the $m$ scheme" or "the zeroorder coupling sheme" in this work) and pointed out a procedure to obtain $S L$-coupling matrix elements by adapting Eissner and Nussbaumer' ${ }^{24}$ algebraic approach. A main goal of Jones' 
formulation is to provide a numerical algorithm for the calculation of mutual spin-orbit matrix elements by digital computers; however, it is not a convenient formulation for the analytical study or calculation of the mutual spin-orbit matrix elements.

In this paper we present an alternative form for the mutual spin-orbit interaction operator in terms of products of orbital and spin angularmomentum operators. Because wave functions of the zero-order coupling scheme are usually eigenfunctions of orbital and spin angular momenta, this form yields matrix elements in a straightforward manner. The most general matrix element of the mutual spin-orbit interaction is obtained by this method and is then presented in a graphical form. This graphical form permits one to calculate the mutual spin-orbit matrix element in any coupling scheme for arbitrary configurations by standard graphical techniques. ${ }^{25-27}$ While in all cases both analytical and graphical forms are presented, we do gain in the graphical form some physical insight, similar to the visual understanding of physical processes provided by Feynman diagrams. Moreover, the graphical formulation gives a transparent representation of the angular-momentum selection rules. Although by today's electronic computers it is possible to manipulate complicated analytical expressions, ${ }^{3}$ the present graphical approach furnishes a means to obtain analy tical results from easily constructed diagrams.

In Sec. II we derive the desired angular-momentum form for the mutual spin-orbit interaction. In Sec. III we obtain the general matrix element in the $m$ scheme and introduce its graphical counterpart. In Sec. IV the procedure is given for computing the matrix element between arbitrary $L S$-coupled configurations using the graphical formulation of $\mathrm{El} \mathrm{Baz}$ and $\mathrm{Castel}^{26}$ and the covariant notations ${ }^{28}$ for Wigner's $3-j$ symbol. The procedure is illustrated for interacting configurations having two electrons outside closed shells. In Sec. $\mathrm{V}$ the procedure is given for computing the matrix element between arbitrary LS-coupled configurations using the graphical formulation of Briggs. ${ }^{27}$ In Sec. VI the trivial case of spin-orbit interaction in a central field is treated for completeness. The physical significance of the mutual spin-orbit interaction is reviewed in Sec. VII, where some commonly used terminologies are explicitly defined, and their graphical representations given. Also in Sec. VII the reduction of two-particle spin-orbit operators to one-particle spin-orbit operators for certain cases is demonstrated graphically, and the spinorbit parameter corresponding to Blume and Watson's ${ }^{17} \zeta_{c}$ is calculated. Appendices A and B sum- marize the graphical rules used in this paper. Appendix C summarizes the results for the matrix element obtained in Sec. IV in the special case of interacting configurations having two electrons outside closed shells. Comparison is also made with certain previously tabulated results ${ }^{13,21(a), 21(b)}$ in particular cases.

\section{SPIN-ORBIT INTERACTIONS IN THE PAULI APPROXIMATION}

As mentioned in the beginning of Sec. I, the quantum electrodynamic interaction between electrons to the lowest order in $\alpha$ can be approximated by the Breit interaction, and the Breit interaction gives rise to the mutual spin-orbit interaction, among others, in the Pauli approximation. Consequently, the total spin-orbit interaction for many-electron atoms can be written, in atomic units, as ${ }^{10}$

$$
V_{s o}=\frac{\alpha^{2}}{2} Z \sum_{i=1}^{N} \frac{1}{r_{i}^{3}} \overrightarrow{\mathrm{L}}_{i} \cdot \overrightarrow{\mathrm{s}}_{i}+\sum_{i \neq j}^{N} V_{i j}
$$

and

$$
V_{i j}=-\frac{\alpha^{2}}{2}\left[\frac{\left(\overrightarrow{\mathrm{r}}_{i}-\overrightarrow{\mathrm{r}}_{j}\right)}{r_{i j}^{3}} \times \overrightarrow{\mathrm{p}}_{i}\right] \cdot\left(\overrightarrow{\mathrm{s}}_{i}+2 \overrightarrow{\mathrm{s}}_{j}\right),
$$

where $\alpha$ is the fine-structure constant, $Z$ the nuclear charge, and $N$ the number of electrons. Here the first term on the right-hand side of (3) accounts for the spin-orbit interaction of each electron in the field of the nucleus, and the second term arises from the mutual spin-orbit interaction between electrons, including the spin-selforbit and spin-other-orbit contributions. Blume and Watson ${ }^{17}$ have derived a tensor-operator form for the mutual spin-orbit interaction (4), but we find, however, an alternative form in terms of products of spin and orbital angular-momentum operators. Because wave functions of the zeroorder coupling scheme are usually eigenfunctions of spin and orbital angular momenta, the latter form will yield the matrix element in a straightforward manner. By inspecting the mutual spinorbit interaction in Eq. (4), we see that the spatial part has the form $\vec{\nabla}_{1}\left(1 / r_{12}\right) \times \vec{\nabla}_{1}$. We can rewrite this as

$$
\begin{aligned}
\vec{\nabla}_{1}\left(\frac{1}{r_{12}}\right) \times \vec{\nabla}_{1}= & \frac{1}{r_{1}^{2}}\left[\overrightarrow{\mathrm{r}}_{1} \times \vec{\nabla}_{1}\left(\frac{1}{r_{12}}\right)\right] \times\left(\overrightarrow{\mathrm{r}}_{1} \times \vec{\nabla}_{1}\right) \\
& -\frac{1}{r_{1}}\left[\overrightarrow{\mathrm{r}}_{1} \times \vec{\nabla}_{1}\left(\frac{1}{r_{12}}\right)\right] \frac{\partial}{\partial r_{1}} \\
& +\frac{1}{r_{1}}\left[\frac{\partial}{\partial r_{1}}\left(\frac{1}{r_{12}}\right)\right]\left(\overrightarrow{\mathrm{r}}_{1} \times \vec{\nabla}_{1}\right),
\end{aligned}
$$

which may be derived by utilizing the identities 


$$
\begin{aligned}
& \overrightarrow{\mathrm{A}} \times(\overrightarrow{\mathrm{B}} \times \overrightarrow{\mathrm{C}})=\overrightarrow{\mathrm{B}}(\overrightarrow{\mathrm{A}} \cdot \overrightarrow{\mathrm{C}})-(\overrightarrow{\mathrm{A}} \cdot \overrightarrow{\mathrm{B}}) \overrightarrow{\mathrm{C}}, \\
& \overrightarrow{\mathrm{A}} \times(\overrightarrow{\mathrm{B}} \times \overrightarrow{\mathrm{C}})+\overrightarrow{\mathrm{B}} \times(\overrightarrow{\mathrm{C}} \times \overrightarrow{\mathrm{A}})+\overrightarrow{\mathrm{C}} \times(\overrightarrow{\mathrm{A}} \times \overrightarrow{\mathrm{B}})=0 .
\end{aligned}
$$

Hence we can decompose the mutual spin-orbit interaction into three terms,

$$
\begin{aligned}
V_{12} & =-\frac{\alpha^{2}}{2}\left[\frac{\left(\overrightarrow{\mathrm{r}}_{1}-\overrightarrow{\mathrm{r}}_{2}\right)}{r_{12}^{3}} \times \overrightarrow{\mathrm{p}}_{1}\right] \cdot\left(\overrightarrow{\mathrm{s}}_{1}+2 \overrightarrow{\mathrm{s}}_{2}\right) \\
& =V^{(1)}+V^{(2)}+V^{(3)},
\end{aligned}
$$

where

$$
\begin{aligned}
& V^{(1)}=i \frac{\alpha^{2}}{2} \frac{1}{r_{1}^{2}}\left[\overrightarrow{\mathrm{L}}_{1}\left(\frac{1}{r_{12}}\right)\right] \times \overrightarrow{\mathrm{L}}_{1} \cdot\left(\overrightarrow{\mathrm{s}}_{1}+2 \overrightarrow{\mathrm{s}}_{2}\right), \\
& V^{(2)}=-\frac{\alpha^{2}}{2} \frac{1}{r_{1}}\left[\overrightarrow{\mathrm{L}}_{1}\left(\frac{1}{r_{12}}\right)\right] \frac{\partial}{\partial r_{1}} \cdot\left(\overrightarrow{\mathrm{s}}_{1}+2 \overrightarrow{\mathrm{s}}_{2}\right), \\
& V^{(3)}=\frac{\alpha^{2}}{2} \frac{1}{r_{1}}\left[\frac{\partial}{\partial r_{1}}\left(\frac{1}{r_{12}}\right)\right] \overrightarrow{\mathrm{L}}_{1} \cdot\left(\overrightarrow{\mathrm{s}}_{1}+2 \overrightarrow{\mathrm{s}}_{2}\right) .
\end{aligned}
$$

Here $\overrightarrow{\mathrm{L}}_{1}=-i \overrightarrow{\mathrm{r}}_{1} \times \vec{\nabla}_{1}$ is the orbital angular-momentum operator of electron 1 . It is worth noting that $V_{12} \neq V_{21}$, and that the total mutual spin-orbit interaction between electrons 1 and 2 is $V(12)$ $=V_{12}+V_{21}$, which is symmetric with respect to electrons 1 and 2.

\section{GENERAL MATRIX ELEMENT IN THE $\boldsymbol{m}$ SCHEME}

Matrix elements of a two-particle operator in any many-particle configuration can always be expressed as linear combinations of matrix elements in corresponding two-particle configurations. ${ }^{29}$ We therefore deal first with the general matrix element for two-particle configurations in the $m$ scheme,

$$
\begin{aligned}
& \left\langle a b\left|V_{12}\right| c d\right\rangle \\
& \equiv\left\langle\phi_{n_{a} l_{a} m_{a} \mu_{a}}(1) \phi_{n_{b} l_{b} m_{b} \mu_{b}}(2)\right| V^{(1)}+V^{(2)}+V^{(3)} \\
& \times\left|\phi_{n_{c} c^{l} c^{m} c^{\mu} c}(1) \phi_{n_{d^{l} d^{m} d^{\mu}{ }_{d}}}(2)\right\rangle \text {, }
\end{aligned}
$$

where $n_{a}$ denotes the principal quantum number, $l_{a}$ the orbital angular-momentum quantum number, and $m_{a}$ and $\mu_{a}$ are the magnetic quantum numbers of the orbital and spin angular momenta, respec- tively, etc. The orbitals have the explicit form

$$
\phi_{n l m \mu}(i)=R_{n l}\left(r_{i}\right) Y_{l m}\left(\Omega_{i}\right) \chi_{\mu}(i) \text {, }
$$

where $\chi_{\mu}$ denotes the spin eigenfunction with $s_{z}=\mu$. To evaluate the matrix element (7), we note that vector spherical harmonics may be generated from scalar spherical harmonics ${ }^{30,31}$ by the use of the orbital angular-momentum operator

$$
\begin{aligned}
\overrightarrow{\mathrm{L}} Y_{l m} & =[l(l+1)]^{1 / 2} \overrightarrow{\mathrm{Y}}_{l l m} \\
& =[l(l+1)]^{1 / 2} \sum_{q} c(l 1 l ; m-q, q) Y_{l(m-q)} \hat{e}_{q}
\end{aligned}
$$

and similarly,

$$
\vec{s} \chi_{\mu}=\left[\frac{1}{2}\left(\frac{1}{2}+1\right)\right]^{1 / 2} \sum_{q} C\left(\frac{1}{2} 1 \frac{1}{2} ; \mu-q, q\right) \chi_{\mu-q} \hat{e}_{q} .
$$

Here $C(l 1 l ; m-q, q)$, and $C\left(\frac{1}{2} 1 \frac{1}{2} ; \mu-q, q\right)$ are ClebschGordan coefficients, and $\hat{e}_{\alpha}$ are spherical unit vectors defined in terms of Cartesian unit vectors by

$$
\begin{aligned}
& \hat{e}_{+1}=-(1 / \sqrt{2})\left(\hat{e}_{x}+i \hat{e}_{y}\right), \\
& \hat{e}_{0}=\hat{e}_{z}, \\
& \hat{e}_{-1}=(1 / \sqrt{2})\left(\hat{e}_{x}-i \hat{e}_{y}\right) .
\end{aligned}
$$

Making use of (10), we can easily obtain the spin part of the matrix element (7),

$$
\begin{aligned}
& \left\langle\chi_{\mu_{a}}(1) \chi_{\mu_{b}}(2)\left|\vec{s}_{1}+2 \vec{s}_{2}\right| \chi_{\mu_{c}}(1) \chi_{\mu_{d}}(2)\right\rangle \\
& \quad=\delta_{\mu_{b} \mu_{d}}(\sqrt{3} / 2) C\left(s_{a} 1 s_{c} ; \mu_{a}, \mu_{c}-\mu_{a}\right) \hat{e}_{\mu_{c}-\mu_{a}} \\
& \quad+2 \delta_{\mu_{a} \mu_{c}}(\sqrt{3} / 2) C\left(s_{b} 1 s_{d} ; \mu_{b}, \mu_{d}-\mu_{b}\right) \hat{e}_{\mu_{d^{-}-\mu_{b}}} .
\end{aligned}
$$

Here in order to show the coupling of spin angular momenta explicitly, we use $s_{a}, s_{b}, s_{c}$, and $s_{d}$ instead of their numerical value $\frac{1}{2}$.

For the spatial part, we note that

$$
\frac{1}{r_{12}}=\sum_{l m} \frac{4 \pi}{2 l+1} \frac{r_{l}^{l}}{r_{>}^{l+1}} Y_{l m}\left(\Omega_{1}\right) Y_{l m}^{*}\left(\Omega_{2}\right)
$$

therefore, the angular integration of the electron 2 reduces to

$$
\int d \Omega Y_{l_{b} m_{b}}^{*} Y_{l m}^{*} Y_{l_{d} m_{d}}=\left(\frac{\left(2 l_{b}+1\right)(2 l+1)}{4 \pi\left(2 l_{d}+1\right)}\right)^{1 / 2} C\left(l_{b} i l_{d} ; m_{b}, m_{d}-m_{b}\right) C\left(l_{b} l l_{d} ; 00\right) \delta_{m\left(m_{d}-m_{b}\right)} .
$$

By using (9), we can perform the angular integration of the electron 1 and obtain the results:

(i) For $V^{(1)}$,

$$
\begin{aligned}
i \int d \Omega Y_{l_{a} m_{a}}^{*}\left(\overrightarrow{\mathrm{L}} Y_{l m}\right) \times\left(\overrightarrow{\mathrm{L}} Y_{l_{c} m_{c}}\right)= & -\left(\frac{l(l+1)(2 l+1) l_{c}\left(l_{c}+1\right)\left(2 l_{c}+1\right)}{2 \pi\left(2 l_{a}+1\right)}\right)^{1 / 2} \\
& \times \sum_{\alpha p} C(l 1 l ; m-q, q) C\left(l_{c} 1 l_{c} ; m_{c}-p, p\right) \\
& \times C\left(l_{a} l l_{c} ; m_{a}, m-q\right) C\left(l_{a} l l_{c} ; 00\right) C(111 ; q p) \delta_{m_{a}\left(m-q+m_{c}-p\right)} \hat{e}_{\alpha+p}
\end{aligned}
$$


where we have used

$$
\hat{e}_{q} \times \hat{e}_{p}=i \sqrt{2} C(111 ; q p) \hat{e}_{q+p} \text {, }
$$

which can be derived from the tensor property of the spherical unit vectors.

(ii) For $V^{(2)}$,

$$
\begin{aligned}
\int d \Omega Y_{l_{a} m_{a}}^{*}\left(\overrightarrow{\mathrm{L}} Y_{l m}\right) Y_{l_{c^{m}}}=\left(\frac{l(l+1)(2 l+1)\left(2 l_{c}+1\right)}{4 \pi\left(2 l_{a}+1\right)}\right)^{1 / 2} \sum_{a} C(l 1 l ; m-q, q) C\left(l_{a} l l_{c} ; m_{a}, m-q\right) \\
\quad \times C\left(l_{a} l l_{c} ; 00\right) \delta_{m_{a}\left(m-q+m_{c}\right)} \hat{e}_{q} 。
\end{aligned}
$$

(iii) For $V^{(3)}$,

$$
\begin{aligned}
\int d \Omega Y_{l_{a} m_{a}}^{*} Y_{l m}\left(\overrightarrow{\mathrm{L}} Y_{l_{c^{m}}}\right)=\left(\frac{(2 l+1) l_{c}\left(l_{c}+1\right)\left(2 l_{c}+1\right)}{4 \pi\left(2 l_{a}+1\right)}\right)^{1 / 2} \sum_{p} C\left(l_{c} 1 l_{c} ; m_{c}-p, p\right) C\left(l_{a} l l_{c} ; m_{a} m\right) \\
\times C\left(l_{a} l l_{c} ; 00\right) \delta_{m_{a}\left(m+m_{c}-p\right)} \hat{e}_{p} .
\end{aligned}
$$

Hence it is a simple task to express the $m$ scheme matrix element (7) in terms of radial integrals with the result:

$$
\left\langle a b\left|V_{12}\right| c d\right\rangle=\left\langle a b\left|V^{(1)}\right| c d\right\rangle+\left\langle a b\left|V^{(2)}\right| c d\right\rangle+\left\langle a b\left|V^{(3)}\right| c d\right\rangle,
$$

where

$$
\begin{aligned}
& \left\langle a b\left|V^{(1)}\right| c d\right\rangle=6\left[\left(2 l_{a}+1\right)\left(2 l_{b}+1\right)\left(2 l_{c}+1\right)\left(2 l_{d}+1\right)\right]^{1 / 2}\left[\left(2 l_{c}+1\right) l_{c}\left(l_{c}+1\right)\right]^{1 / 2} \\
& \times(-)^{l_{a}+l_{d}} \sum_{l q p}[(2 l+1) l(l+1)]^{1 / 2}\left(\begin{array}{ccc}
l_{a} & l & l_{c} \\
0 & 0 & 0
\end{array}\right)\left(\begin{array}{ccc}
l_{b} & l & l_{d} \\
0 & 0 & 0
\end{array}\right) R_{l}^{(1)}(a b ; c d) \\
& \times\left(\begin{array}{ccc}
m_{a} & l & l_{c} \\
l_{a} & m_{a}-m_{c}+q & m_{c}-q
\end{array}\right)\left(\begin{array}{ccc}
m_{b} & l_{d} & -m_{b}+m_{d} \\
l_{b} & m_{d} & l
\end{array}\right)\left(\begin{array}{ccc}
l_{c} & q & m_{c}-q \\
m_{c} & 1 & l_{c}
\end{array}\right)\left(\begin{array}{ccc}
l & p & m_{a}-m_{c}+q \\
-m_{b}+m_{d} & 1 & l
\end{array}\right) \\
& \times\left[\delta_{\mu_{b} \mu_{d}}\left(\begin{array}{ccc}
\mu_{a} & 1 & s_{c} \\
s_{a} & \mu_{a}-\mu_{c} & \mu_{c}
\end{array}\right)\left(\begin{array}{ccc}
\mu_{a}-\mu_{c} & 1 & 1 \\
1 & p & q
\end{array}\right)+2 \delta_{\mu_{a} \mu_{c}}\left(\begin{array}{ccc}
\mu_{b} & 1 & s_{d} \\
s_{b} & \mu_{b}-\mu_{d} & \mu_{d}
\end{array}\right)\left(\begin{array}{ccc}
\mu_{b}-\mu_{d} & 1 & 1 \\
1 & q & p
\end{array}\right)\right], \\
& \left\langle a b\left|V^{(2)}\right| c d\right\rangle=-2\left(\frac{3}{2}\right)^{1 / 2}\left[\left(2 l_{a}+1\right)\left(2 l_{b}+1\right)\left(2 l_{c}+1\right)\left(2 l_{d}+1\right)\right]^{1 / 2} \\
& \times(-)^{l_{a}+l_{d}} \sum_{l}[(2 l+1) l(l+1)]^{1 / 2}\left(\begin{array}{ccc}
l_{a} & l & l_{c} \\
0 & 0 & 0
\end{array}\right)\left(\begin{array}{ccc}
l_{b} & l & l_{d} \\
0 & 0 & 0
\end{array}\right) R_{l}^{(2)}(a b ; c d)\left(\begin{array}{ccc}
m_{a} & l_{c} & l \\
l_{a} & m_{c} & m_{a}-m_{c}
\end{array}\right)\left(\begin{array}{ccc}
m_{b} & l_{d} & l \\
l_{b} & m_{d} & m_{b}-m_{d}
\end{array}\right) \\
& \times\left[\delta_{\mu_{b} \mu_{d}}\left(\begin{array}{ccc}
\mu_{a} & 1 & s_{c} \\
s_{a} & \mu_{a}-\mu_{c} & \mu_{c}
\end{array}\right)\left(\begin{array}{ccc}
m_{a}-m_{c} & m_{b}-m_{d} & \mu_{a}-\mu_{c} \\
l & l & 1
\end{array}\right)\right. \\
& \left.+2 \delta_{\mu_{a} \mu_{c}}\left(\begin{array}{ccc}
\mu_{b} & 1 & s_{d} \\
s_{b} & \mu_{b}-\mu_{d} & \mu_{d}
\end{array}\right)\left(\begin{array}{ccc}
m_{a}-m_{c} & m_{b}-m_{d} & \mu_{b}-\mu_{d} \\
l & l & 1
\end{array}\right)\right] \text {, } \\
& \left\langle a b\left|V^{(3)}\right| c d\right\rangle=2\left(\frac{3}{2}\right)^{1 / 2}\left[\left(2 l_{a}+1\right)\left(2 l_{b}+1\right)\left(2 l_{c}+1\right)\left(2 l_{d}+1\right)\right]^{1 / 2}\left[\left(2 l_{c}+1\right) l_{c}\left(l_{c}+1\right)\right]^{1 / 2} \\
& \times(-)^{l_{a}+l_{d}} \sum_{T}\left(\begin{array}{ccc}
l_{a} & l & l_{c} \\
0 & 0 & 0
\end{array}\right)\left(\begin{array}{ccc}
l_{b} & l & l_{d} \\
0 & 0 & 0
\end{array}\right) R_{l}^{(3)}(a b ; c d) \\
& \times\left(\begin{array}{ccc}
m_{a} & l & -m_{a}-m_{b}+m_{d} \\
l_{a}-m_{b}+m_{d} & l_{c}
\end{array}\right)\left(\begin{array}{ccc}
m_{b}-m_{b}+m_{d} & l_{d} \\
l_{b} & l & m_{d}
\end{array}\right) \\
& \times\left[\delta_{\mu_{b} \mu_{d}}\left(\begin{array}{ccc}
\mu_{a} & 1 & s_{c} \\
s_{a} & \mu_{a}-\mu_{c} & \mu_{c}
\end{array}\right)\left(\begin{array}{ccc}
\mu_{a}-\mu_{c} & l_{c} & l_{c} \\
1 & -m_{a}-m_{b}+m_{d} & m_{c}
\end{array}\right)\right. \\
& \left.+2 \delta_{\mu_{a} \mu_{c}}\left(\begin{array}{ccc}
\mu_{b} & 1 & s_{d} \\
s_{b} & \mu_{b}-\mu_{d} & \mu_{d}
\end{array}\right)\left(\begin{array}{ccc}
\mu_{b}-\mu_{d} & l_{c} & l_{c} \\
1 & -m_{a}-m_{b}+m_{d} & m_{c}
\end{array}\right)\right] \text {. }
\end{aligned}
$$

The radial integrals in Eq. (20) are defined by 


$$
\begin{aligned}
& R_{l}^{(1)}(a b ; c d)=\frac{\alpha^{2}}{4} \int_{0}^{\infty} r_{1}^{2} d r_{1} \int_{0}^{\infty} r_{2}^{2} d r_{2} R_{n_{a} l_{a}}\left(r_{1}\right) R_{n_{b} l_{b}}\left(r_{2}\right) \frac{r_{<}^{l}}{r_{>}^{l+1}} \frac{1}{r_{1}^{2}} R_{n_{c} l_{c}}\left(r_{1}\right) R_{n_{d} l_{d}}\left(r_{2}\right), \\
& R_{l}^{(2)}(a b ; c d)=\frac{\alpha^{2}}{4} \int_{0}^{\infty} r_{1}^{2} d r_{1} \int_{0}^{\infty} r_{2}^{2} d r_{2} R_{n_{a} l_{a}}\left(r_{1}\right) R_{n_{b} l_{b}}\left(r_{2}\right) \frac{r_{<}^{l}}{r_{>}^{l+1}} \frac{1}{r_{1}}\left(\frac{\partial}{\partial r_{1}} R_{n_{c} l_{c}}\left(r_{1}\right)\right) R_{n_{d} l_{d}}\left(r_{2}\right), \\
& R_{l}^{(3)}(a b ; c d)=\frac{\alpha^{2}}{4} \int_{0}^{\infty} r_{1}^{2} d r_{1} \int_{0}^{\infty} r_{2}^{2} d r_{2} R_{n_{a} l_{a}}\left(r_{1}\right) R_{n_{b} l_{b}}\left(r_{2}\right) \frac{1}{r_{1}} \frac{\partial}{\partial r_{1}}\left(\frac{r_{\zeta}^{l}}{r_{>}^{l+1}}\right) R_{n_{c} l_{c}}\left(r_{1}\right) R_{n_{d} l_{d}}\left(r_{2}\right),
\end{aligned}
$$

where $r_{>}$and $r_{<}$denote the greater and lesser of $r_{1}$ and $r_{2}$, respectively. Also in Eq. (20), we have replaced all the Clebsch-Gordan coefficients by Wigner's $3-j$ symbols in the covariant notation ${ }^{28}$ to better represent their tensor property and to avoid carrying phase factors. For example, the component of the $3-j$ symbol which is contravariant in the first two indices and covariant in the last is

$$
\left(\begin{array}{lll}
j_{1} & j_{2} & m_{3} \\
m_{1} & m_{2} & j_{3}
\end{array}\right)=(-)^{j_{3}-m_{3}}\left(\begin{array}{ccc}
j_{1} & j_{2} & j_{3} \\
m_{1} & m_{2} & -m_{3}
\end{array}\right) .
$$

Note that this definition of $\mathrm{El} \mathrm{Baz}$ and $\mathrm{Castel}^{26}$ is different from that of Wigner, ${ }^{28}$ who assigns the names "covariant" and "contravariant" inversely.

We can visualize the mutual spin-orbit interaction by presenting its matrix elements graphically. The expression (20a) is represented graphically as

$$
\begin{aligned}
& \left\langle a b\left|V^{(1)}\right| c d\right\rangle=6\left[\left(2 l_{a}+1\right)\left(2 l_{b}+1\right)\left(2 l_{c}+1\right)\left(2 l_{d}+1\right)\right]^{1 / 2}\left[\left(2 l_{c}+1\right) l_{c}\left(l_{c}+1\right)\right]^{1 / 2} \\
& \times(-)^{l_{a}+l_{d}} \sum_{l}[(2 l+1) l(l+1)]^{1 / 2}\left(\begin{array}{ccc}
l_{a} & l & l_{c} \\
0 & 0 & 0
\end{array}\right)\left(\begin{array}{ccc}
l_{b} & l & l_{d} \\
0 & 0 & 0
\end{array}\right) R_{l}^{(1)}(a b ; c d) \\
& \left\{\int_{1}^{l_{a}+l_{c}}\right.
\end{aligned}
$$

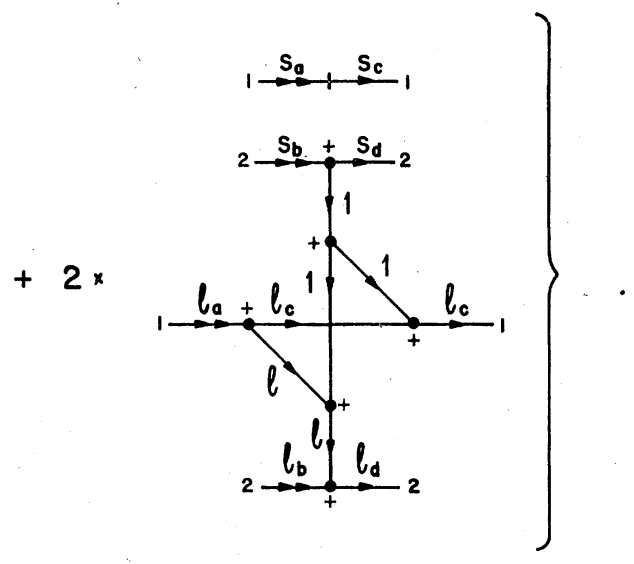

The notational rules used to describe a $3-j$ symbol are as follows:

(i) Each vertex, indicated by a node, represents a 3-j symbol: each contravariant component is

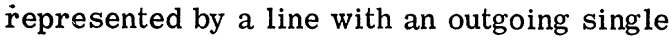
arrow, and each covariant component by a line with an ingoing double arrow.

(ii) The plus (minus) sign at the vertex means that the angular momenta are to be read counterclockwise (clockwise).

The magnetic quantum number of each angularmomentum line is usually suppressed where no confusion may occur. Also, we note that the "current" of magnetic quantum numbers is conserved at each node due to the selection rule for magnetic quantum numbers of the covariant $3-j$ symbol; for example,

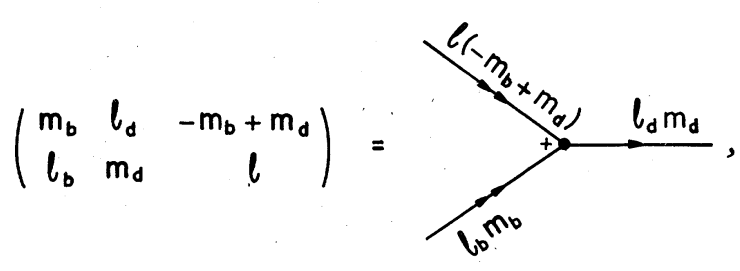

where we have the ingoing current " $m_{b}+\left(-m_{b}+m_{d}\right)$ ") = the outgoing current " $m_{d}$ ".

The notational rules used to combine $3-j$ symbols are as follows:

(i) The summation, or in the tensorial term "contraction", over a pair of magnetic quantum numbers (one of which is always contravariant, and the other covariant) is performed by joining the corresponding angular-momentum lines to form a linked single-arrowed line. 
(ii) The change in direction of a linked angularmomentum line $j$ introduces a phase factor $(-)^{2 j}$. As a result, we may suppress the arrow of a linked angular-momentum line $j$ whenever $j$ is an integer.

Although we do not write a magnetic quantum number for a linked angular-momentum line, the summation over the magnetic quantum number is always implied. Nevertheless, in many cases because of the conservation of the "magnetic current," the summation implied by a linked angular-momentum line exists only formally.

The scalar product of a covariant eigenvector $\left\langle j_{a} m_{a}\right|$ and a contravariant eigenvector $\left|j_{b} m_{b}\right\rangle$ is denoted graphically by

$$
\left\langle j_{a} m_{a} \mid j_{b} m_{b}\right\rangle=\stackrel{j_{a} m_{a},+j_{b} m_{b}}{\longrightarrow}=\delta_{j_{a} j_{b}} \delta_{m_{a} m_{b}} .
$$

The number 1 or 2 at the free end of an open angular-momentum line signifies the electron 1 or 2 , respectively; these electron indices will be suppressed where no confusion may occur.

With the foregoing graphical rules, we can similarly represent $(20 \mathrm{~b})$ and $(20 \mathrm{c})$ by the diagrams

$$
\begin{aligned}
& \left\langle a b\left|V^{(2)}\right| c d\right\rangle=-2\left(\frac{3}{2}\right)^{1 / 2}\left[\left(2 l_{a}+1\right)\left(2 l_{b}+1\right)\left(2 l_{c}+1\right)\left(2 l_{d}+1\right)\right]^{1 / 2} \\
& \times(-)^{l_{a}+l_{d}} \sum_{l}[(2 l+1) l(l+1)]^{1 / 2}\left(\begin{array}{ccc}
l_{a} & l & l_{c} \\
0 & 0 & 0
\end{array}\right)\left(\begin{array}{ccc}
l_{b} & l & l_{d} \\
0 & 0 & 0
\end{array}\right) R_{l}^{(2)}(a b ; c d) .
\end{aligned}
$$

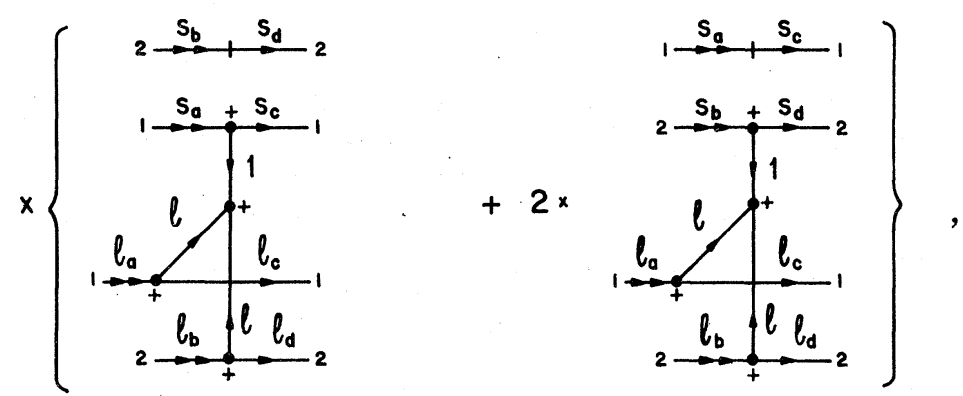

$$
\begin{aligned}
& \left\langle a b\left|V^{(3)}\right| c d\right\rangle=2\left(\frac{3}{2}\right)^{1 / 2}\left[\left(2 l_{a}+1\right)\left(2 l_{b}+1\right)\left(2 l_{c}+1\right)\left(2 l_{d}+1\right)\right]^{1 / 2}\left[\left(2 l_{c}+1\right) l_{c}\left(l_{c}+1\right)\right]^{1 / 2} \\
& \times(-)^{l_{a}+l_{d}} \sum_{l}\left(\begin{array}{ccc}
l_{a} & l & l_{c} \\
0 & 0 & 0
\end{array}\right)\left(\begin{array}{ccc}
l_{b} & l & l_{d} \\
0 & 0 & 0
\end{array}\right) R_{l}^{(3)}(a b ; c d)
\end{aligned}
$$

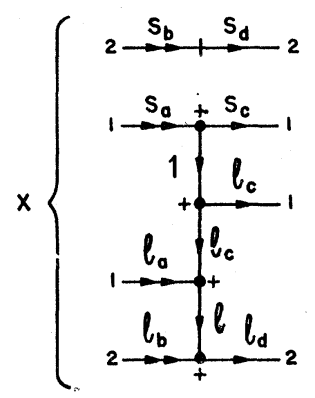

$$
\begin{aligned}
& +2 \times \quad \underbrace{l_{b}}_{+}
\end{aligned}
$$

To facilitate the calculation of the matrix element in different coupling schemes, we find that the expressions (20a), (20b), and (20c) can be rearranged into a sum of similar diagrams. The rearrangement can be easily carried out by graphical transformation rules presented in Appendix A. For example, in the first diagram in (22c) we apply rule (A5) to the open angular-momentum lines $l_{a}$ and $l_{c}$ and the linked angularmomentum lines 1 and $l$. As a result, we obtain a sum of products of an open diagram similar to those in (22b) and a closed diagram which is just the graphical representation of a 6-j symbol. The second diagram in (22c) and both diagrams in (22a) can be reduced in a similar way to diagrams of the form of those in (22b). Thus by such procedures, we can rewrite (19) as

$$
\left\langle a b\left|V_{12}\right| c d\right\rangle=\sum_{l k} Q_{l k}(a b ; c d) G_{l k}(a b ; c d) .
$$

The graphical portion $G_{l k}(a b ; c d)$ is 


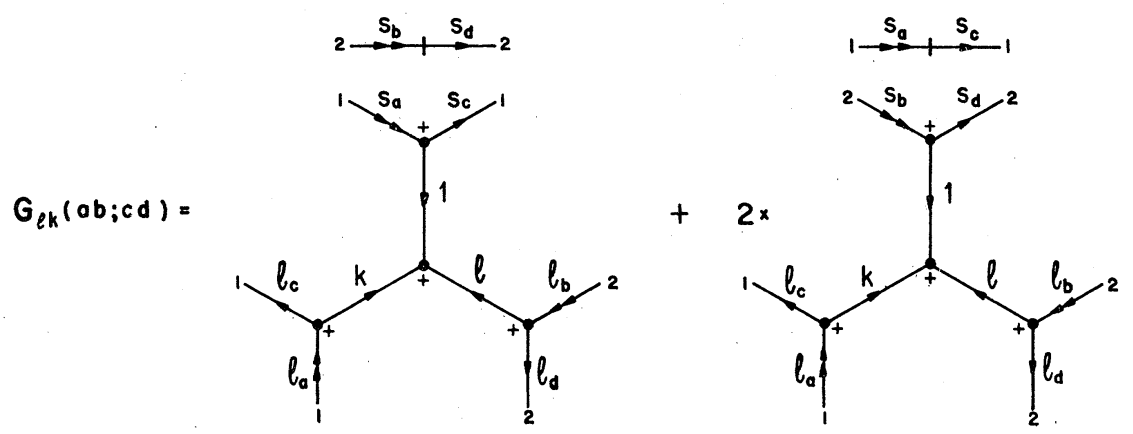

or, analytically,

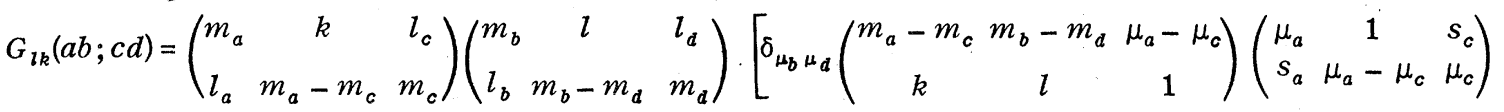

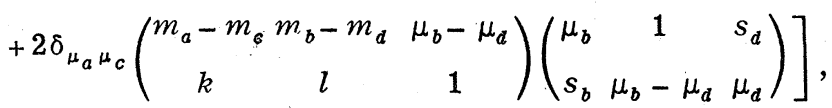

where all the summations over magnetic quantum numbers are eliminated due to the conservation of the "magnetic current" although we may formally reinstate the summations as implicitly represented by the diagram (24). The coefficient $Q_{l_{k}}(a b ; c d)$ in (23) is

$$
\begin{aligned}
Q_{l k}(a b ; c d)= & 2\left(\frac{3}{2}\right)^{1 / 2}\left[\left(2 l_{a}+1\right)\left(2 l_{b}+1\right)\left(2 l_{c}+1\right)\left(2 l_{d}+1\right)\right]^{1 / 2}(-)^{l_{a}+l_{d}}\left(\begin{array}{lll}
l_{a} & l & l_{c} \\
0 & 0 & 0
\end{array}\right)\left(\begin{array}{ccc}
l_{b} & l & l_{d} \\
0 & 0 & 0
\end{array}\right) \\
& \times\left[-\delta_{l k}[(2 l+1) l(l+1)]^{1 / 2} R_{l}^{(2)}(a b ; c d)+\left[\left(2 l_{c}+1\right) l_{c}\left(l_{c}+1\right)\right]^{1 / 2}(2 k+1)\left\{\begin{array}{lll}
l_{c} & l & l_{a} \\
k & l_{c} & 1
\end{array}\right\} I_{l_{k}}(a b ; c d)\right],
\end{aligned}
$$

where the radial integral $I_{l k}(a b ; c d)$ is defined as

$$
I_{l k}(a b ; c d)=\frac{1}{2}[l(l+1)-k(k+1)+2] R_{l}^{(1)}(a b ; c d)+R_{l}^{(3)}(a b ; c d) .
$$

If we represent the factor $Q_{l k}(a b ; c d)$ by a circle about the vertex involving angular momenta $(k l 1)$, then the general matrix element (23) of the mutual spin-orbit interaction in the $m$ scheme can be graphically represented in the simple form

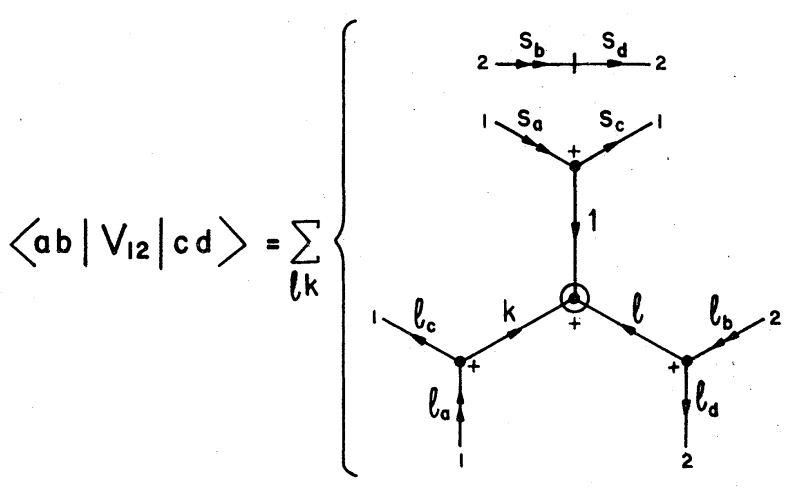

IV. MATRIX OF SPIN-ORBIT INTERACTION IN THE LSJM SCHEME

By using the form (28) for the general matrix element of the mutual spin-orbit interaction in the

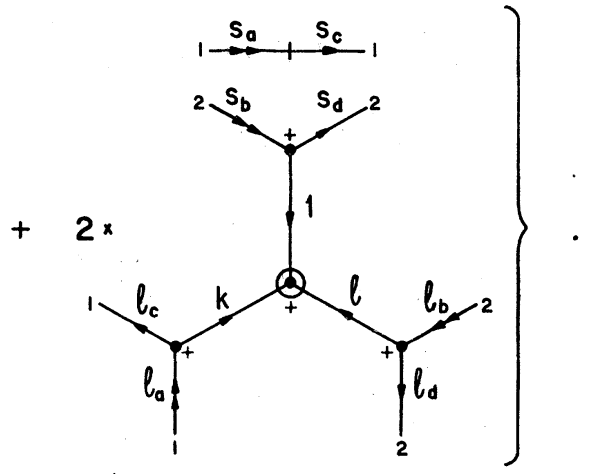

$m$ scheme, the calculation of these matrix elements for arbitrary configurations in any coupling scheme can be carried out easily by the graphical method. Here, as an example, we will treat the particular case of configurations having two non- 
equivalent electrons outside any number of closed shells in $L S$-coupling. The extension to the cases of equivalent electrons is given in Appendix C. Calculation of the mutual spin-orbit matrix elements for other configurations by the graphical method requires the construction of the appropriate configuration diagram. Appropriate configuration diagrams, for use with our mutual spin-orbit diagram (28), may be constructed using the techniques of El Baz and Castel. ${ }^{26}$ An alternative form of the spin-orbit diagram (28), appropriate for use with configuration diagrams constructed by Briggs ${ }^{27}$ techniques, is given in the next section.

In terms of $m$ scheme eigenstates, the LS-coupled state for two nonequivalent electrons is represented by

$$
\begin{aligned}
\left|\phi_{a b}(12)\right\rangle & \equiv\left|\left(l_{a} l_{b}\right) L\left(s_{a} s_{b}\right) S J M\right\rangle \\
& =\sum_{m_{a}^{m_{a}} \mu_{b}^{m_{b}}} C^{m_{a} m_{b} \mu_{a} \mu_{b}}|a b\rangle .
\end{aligned}
$$

Here $|a b\rangle$ are the $m$-scheme eigenstates

$$
\begin{aligned}
|a b\rangle & \equiv\left|\phi_{n_{a} l_{a} m_{a} \mu_{a}}(1) \phi_{n_{b} l_{b} m_{b} \mu_{b}}(2)\right\rangle \\
& =\left|l_{a} m_{a}(1)\right\rangle\left|s_{a} \mu_{a}(1)\right\rangle\left|l_{b} m_{b}(2)\right\rangle\left|s_{b} \mu_{b}(2)\right\rangle, .
\end{aligned}
$$

and the expansion coefficients $C^{m_{a} m_{b} \mu_{a} \mu_{b}}$ are

$$
\begin{aligned}
C^{m_{a} m_{b} \mu_{a} \mu_{b}=} & -[(2 S+1)(2 L+1)(2 J+1)]^{1 / 2} \\
& \times \sum_{m_{L} m_{S}}\left(\begin{array}{lll}
m_{a} & L & m_{b} \\
l_{a} & m_{L} & l_{b}
\end{array}\right)\left(\begin{array}{ccc}
\mu_{a} & S & \mu_{b} \\
s_{a} & m_{S} & s_{b}
\end{array}\right) \\
& \times\left(\begin{array}{ccc}
m_{L} & J & m_{S} \\
L & M & S
\end{array}\right)
\end{aligned}
$$

The $L S$-coupled state (29) can be represented in graphical form in a number of ways, but the two common forms are those of $\mathrm{El} \mathrm{Baz}$ and Castel $\mathrm{Cl}^{26}$ and of Briggs. ${ }^{27}$ In this paper we propose to use a modified form of $\mathrm{El} \mathrm{Baz}$ and Castel's notation, ${ }^{26}$ i.e., we employ 3-j diagrams to construct a configuration diagram, rather than using ClebschGordan coefficient diagrams, and use a bar on an angular-momentum symbol $j$ (i.e., $\bar{j}$ ) to indicate the multiplication factor $(2 j+1)^{1 / 2}$ thus incurred.

We represent, then, the $L S$-coupled state (29) by the diagram

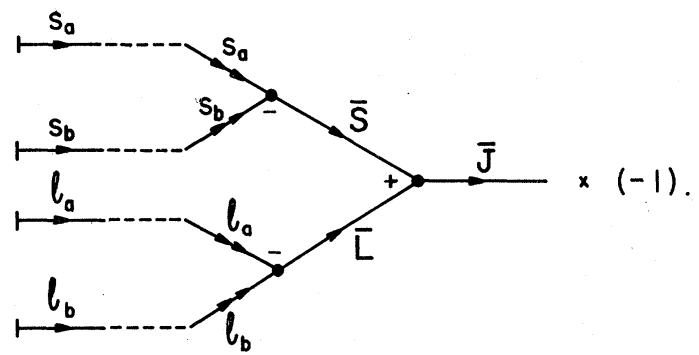

In (32) a bar on an angular-momentum symbol $j$ indicates a multiplication factor $(2 j+1)^{1 / 2}$. The contravariant vectors (or ket vectors) $\left|l_{a} m_{a}(1)\right\rangle$, $\left|s_{a^{\prime}} \mu_{a}(1)\right\rangle$, etc. are each denoted by a vertical bar with an outgoing single arrow. The dotted lines indicate the linkage of the eigenstate $|a b\rangle$ with the angular-momentum coupling coefficient $C^{m} a^{m}{ }_{b} \mu_{a} \mu_{b}$; this corresponds to the summation over the magnetic quantum numbers $m_{a}, m_{b}, \mu_{a}$, and $\mu_{b}$ in the analytical version (29) of the $L S$-coupled state.

The conjugate $L S$-coupled state is represented by

$$
\begin{aligned}
\left\langle\phi_{a b}(12)\right|= & \sum_{\substack{m_{a} m_{b} m_{b} \\
\mu_{b}}} C_{m_{a} m_{b} \mu_{a} \mu_{b}}\langle a b| \\
= & -\sum_{\substack{m_{a}^{a} m_{b} \\
\mu_{b}}}[(2 S+1)(2 L+1)(2 J+1)]^{1 / 2} \\
& \quad \times \sum_{m_{L} m_{s}}\left(\begin{array}{ccc}
c_{a} & m_{L} & l_{b} \\
m_{a} & L & m_{b}
\end{array}\right)\left(\begin{array}{ccc}
s_{a} & m_{s} & s_{b} \\
\mu_{a} & S & \mu_{b}
\end{array}\right)\left(\begin{array}{ccc}
L & M & S \\
m_{L} & J & m_{s}
\end{array}\right)\left\langle l_{a} m_{a}(1)\right|\left\langle s_{a} \mu_{a}(1)\right|\left\langle l_{b} m_{b}(2)\right|\left\langle s_{b} \mu_{b}(2)\right|,
\end{aligned}
$$

or graphically by

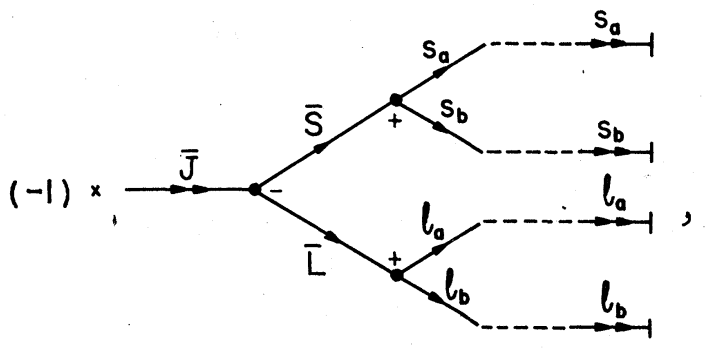

where the vertical bars with an ingoing double ar- row denote covariant vectors (or bra vectors).

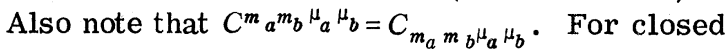
shells, we can simply use the $m$-scheme eigenstates $\left|l_{i} m_{i}\right\rangle\left|s_{i} \mu_{i}\right\rangle$ and $\left\langle l_{i} m_{i}\right|\left\langle s_{i} \mu_{i}\right|$, i.e.,

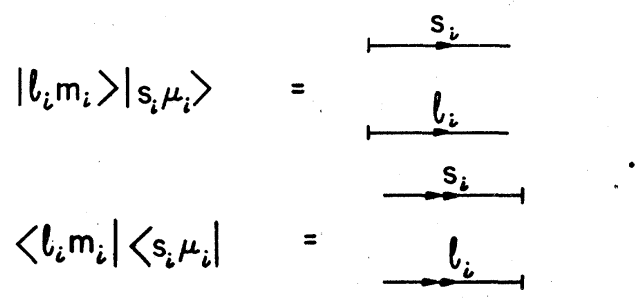


Using the graphical expressions (32) and (34) to represent the $L S$-coupled states of two nonequivalent electrons and using (35) to represent the closed-shell electron states, we employ (28) to evaluate matrix elements of the mutual spin-orbit interaction between configurations having two nonequivalent electrons outside closed shells. These matrix elements are conveniently divided into three categories: the interaction between the outer electrons, the interaction between the outer - and closed-shell electrons, and the interaction between the closed-shell electrons. In what follows, we consider each of these in turn.

A. Mutual spin-orbit interaction between the outer electrons

For any two-particle operator, the interacting configurations can differ at most by two orbitals.
Therefore, the most general matrix element in this case involves four different outer electron orbitals, i.e., $\left\langle\phi_{a b}(12)\left|V_{12}\right| \phi_{c d}^{\prime}(12)\right\rangle$, where $\left|\phi_{c d}^{\prime}(12)\right\rangle$ $\equiv\left|\left(l_{c} l_{d}\right) L^{\prime}\left(s_{c} s_{d}\right) S^{\prime} J M\right\rangle$. This matrix element can be expressed as a linear combination of $m$-scheme matrix elements, i.e.,

$$
\begin{aligned}
& \left\langle\phi_{a b}(12)\left|V_{12}\right| \phi_{c d}^{\prime}(12)\right\rangle \\
& \quad=\sum_{\text {all } m \text { and } \mu} C_{m_{a} m_{b} \mu_{a} \mu_{b}}\left\langle a b\left|V_{12}\right| c d\right\rangle C^{\prime m_{c} m_{d} \mu_{c} \mu_{d}} .
\end{aligned}
$$

Graphically, the summation over magnetic quantum numbers in the matrix element (36) can be carried out by joining corresponding angular-momentum lines of the diagrams

$\left\langle\phi_{a b}(12)\left|V_{12}\right| \phi_{c d}^{\prime}(12)\right\rangle$

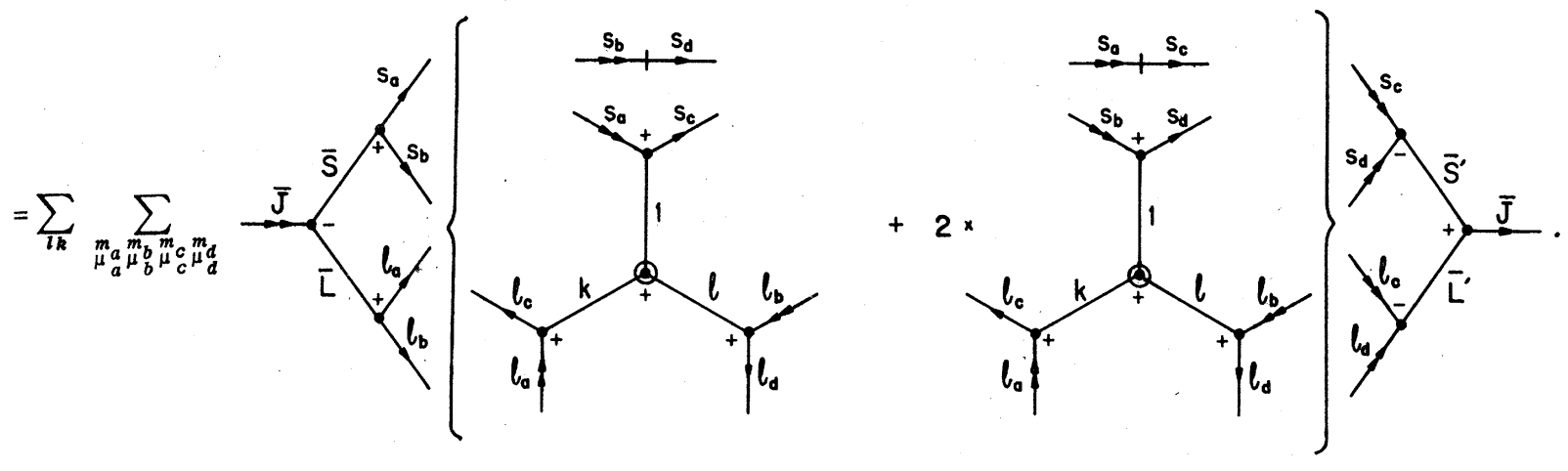

This procedure yields
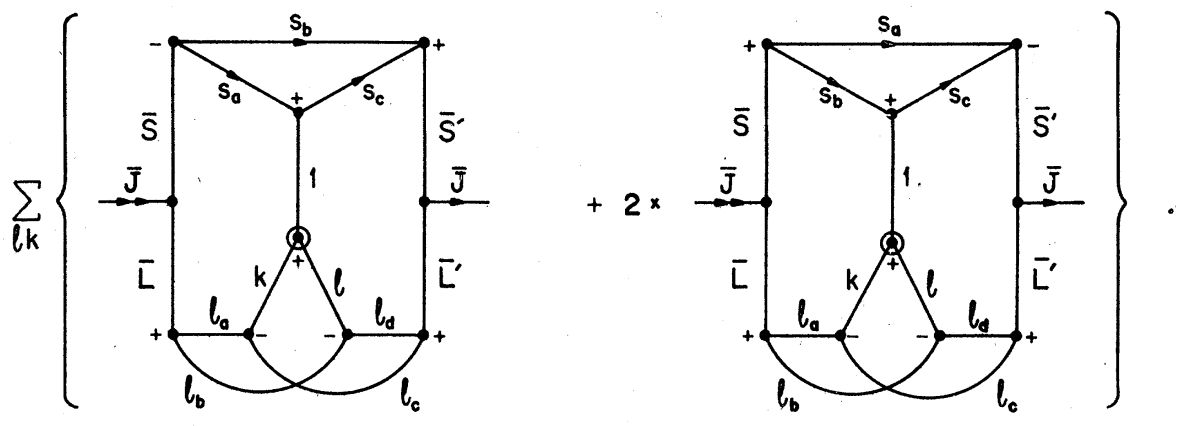
To evaluate these diagrams, we first join together the remaining covariant and contravariant angular-momentum lines $J$ and divide them by the factor $(2 J+1)$ since values of the diagrams are independent of the total magnetic quantum number $M$. This is represented by the graphical transformation rule (A2) in Appendix A. Next we use the graphical transformation rule (A4) to separate the diagrams into

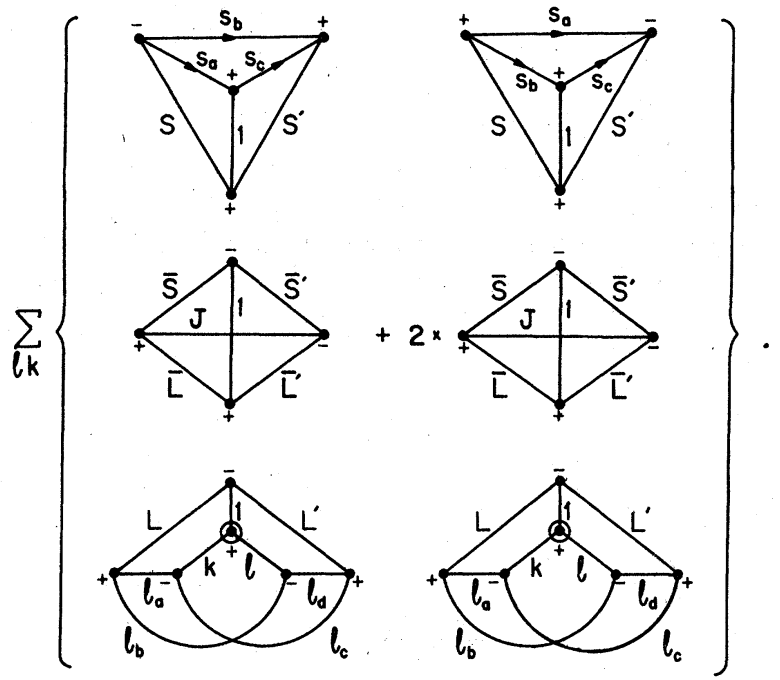

(39)
These are recognizable as basic diagrams whose analytical values are given in Appendix B. By substituting the analytical value for each basic diagram in (39), we obtain the general matrix element in $L S$ coupling for two nonequivalent electrons,

$$
\begin{aligned}
&\left\langle\phi_{a b}(12)\right| V_{12}\left|\phi_{c d}^{\prime}(12)\right\rangle \\
&=\sum_{l k} Q_{l k}(a b ; c d)(-)^{J+L^{\prime}+k^{+}+}\left[2+(-)^{S+S^{\prime}}\right] \\
& \times {\left[(2 L+1)\left(2 L^{\prime}+1\right)(2 S+1)\left(2 S^{\prime}+1\right)\right]^{1 / 2} } \\
& \times\left\{\begin{array}{lll}
S & S^{\prime} & 1 \\
\frac{1}{2} & \frac{1}{2} & \frac{1}{2}
\end{array}\right\}\left\{\begin{array}{lll}
S & L & J \\
L^{\prime} & S^{\prime} & 1
\end{array}\right\}\left\{\begin{array}{lll}
l_{a} & L & l_{b} \\
k & 1 & l \\
l_{c} & L^{\prime} & l_{d}
\end{array}\right\} \\
& \equiv D(a b ; c d) .
\end{aligned}
$$

Since $a, b, c$, and $d$ represent arbitrary orbitals, the calculation of direct and exchange terms is implied.

Now we denote the antisymmetrized $L S$-coupling state of the whole atom by $\left|\phi_{a b}(12) \phi_{\text {closed }}\right\rangle_{A}$, where $\phi_{\text {closed }}$ represents the normalized state of any number of closed shells, and the subscript $A$ outside the ket signifies the antisymmetrization. Therefore, the general matrix element of the mutual spin-orbit interaction between the two nonequivalent electrons outside closed shells is

$$
\left\langle\phi_{a b}(12) \phi_{\text {closed }}\left|\sum_{i \neq j}^{\text {open }} V_{i j}\right| \phi_{c d}^{\prime}(12) \phi_{\text {closed }}\right\rangle_{A}=\left\langle\phi_{a b}(12)\left|V_{12}\right| \phi_{c d}^{\prime}(12)\right\rangle_{A}+\left\langle\phi_{a b}(12)\left|V_{21}\right| \phi_{c d}^{\prime}(12)\right\rangle_{A},
$$

where the summation is only over the outer electron pairs, and the subscript $A$ indicates that the matrix element is taken between the antisymmetrized states. To calculate matrix elements of a two-particle operator between antisymmetrized states considered here we use the following simple two-step procedure: We first evaluate the matrix element using unsymmetrized states. Then we replace each two-particle matrix element by the difference of the direct and exchange terms. Thus, the antisymmetrization can be carried out only in the last step of evaluating the matrix elements with two-particle configurations. Consequently, we can express (41) in terms of (40) as follows:

$$
\begin{aligned}
& \left\langle\phi_{a b}(12) \phi_{\text {closed }}\left|\sum_{i \neq j}^{\text {open }} V_{i j}\right| \phi_{c d}^{\prime}(12) \phi_{\text {closed }}\right\rangle_{A}=\left\langle\phi_{a b}(12)\left|V_{12}\right| \phi_{c d}^{\prime}(12)\right\rangle-\left\langle\phi_{a b}(12)\left|V_{12}\right| \phi_{c d}^{\prime}(21)\right\rangle+\left\langle\phi_{a b}(12)\left|V_{21}\right| \phi_{c d}^{\prime}(12)\right\rangle \\
& -\left\langle\phi_{a b}(12)\left|V_{21}\right| \phi_{c d}^{\prime}(21)\right\rangle \\
& =D(a b ; c d)+(-)^{l_{c}+l_{d^{+}}+L^{\prime}+S^{\prime}} D(a b ; d c)+(-)^{L+S+L^{\prime}+S^{\prime}} D(b a ; d c) \\
& +(-)^{l_{a}+l_{b}+L+s} D(b a ; c d) \text {. }
\end{aligned}
$$

The first and third terms of (42) arise from the direct contribution, and the second and fourth terms arise from the exchange contribution.

\section{B. Mutual spin-orbit interaction between the outer electrons and the closed-shell electrons}

For the interaction between the outer and closed-shell electrons, the two interacting configurations can differ at most by one orbital. However, due to the coupling between the outer electrons, the matrix element will in general involve four different orbitals for the case of two nonequivalent electrons outside closed shells. The total mutual spin-orbit interaction between a pair of electrons is $V(12)=V_{12}+V_{21}$, and 
for each term there are direct and exchange contributions. Thus there are four different types of matrix elements, which we consider in turn:

(i) $\left\langle\phi_{a b}(13) \phi_{i}(2)\left|V_{12}\right| \phi_{c b}^{\prime}(13) \phi_{i}(2)\right\rangle$

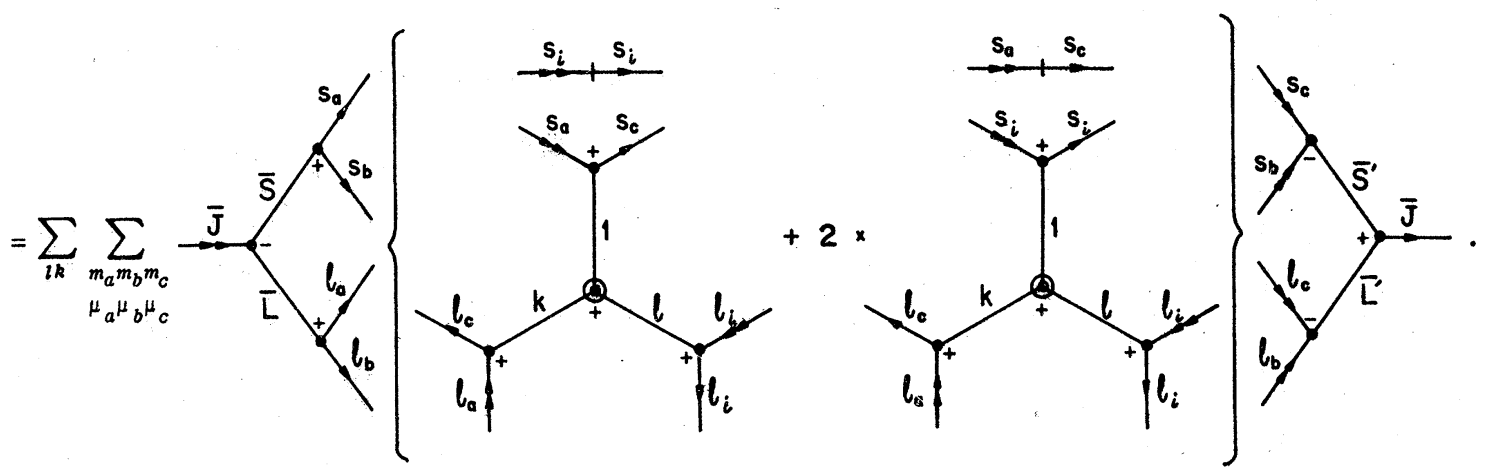

Here $\phi_{i}(2)$ is any $m$-scheme eigenstate belonging to closed shells. The summation over magnetic quantum numbers in (43) is carried out by joining corresponding angular-momentum lines, as stated in the last subsection. In addition, we sum over the closed subshell $i$ by joining the corresponding contravariant and covariant lines of $l_{i}$ and $s_{i}$. Hence, the result of summing the first term of (43) over the closed subshell $i$ is

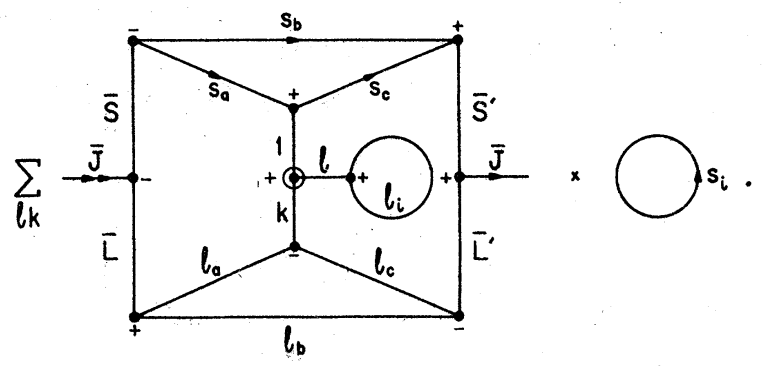

(ii) $\left\langle\phi_{a b}(13) \phi_{i}(2)\left|V_{21}\right| \phi_{c b}^{\prime}(13) \phi_{i}(2)\right\rangle$
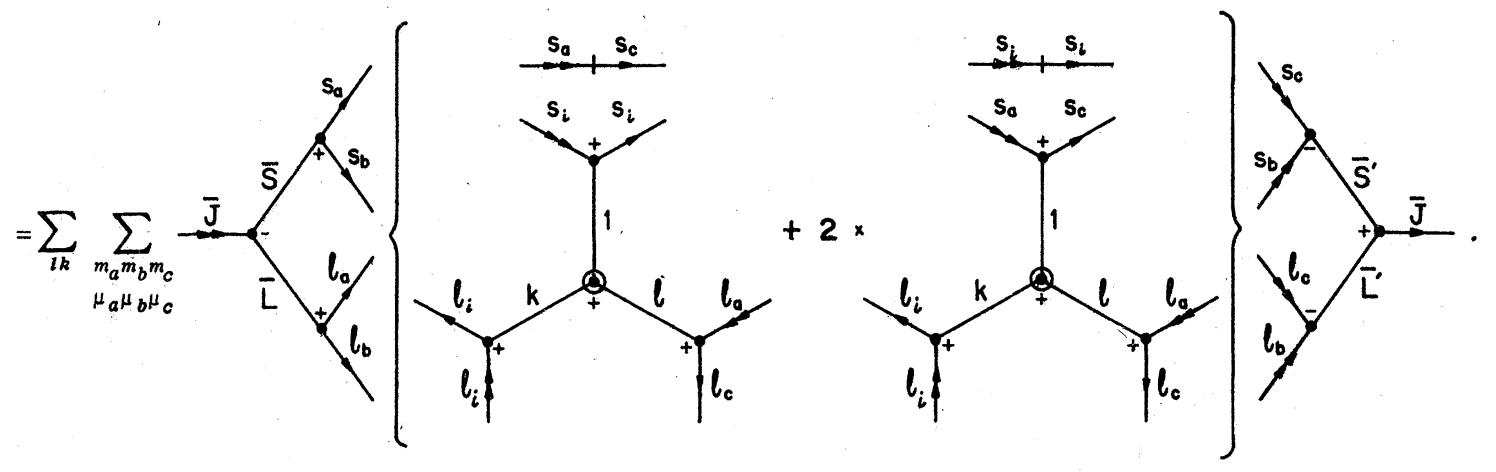
These graphs give zero contribution after summing over closed shells. The first term results in a diagram similar to (46) after connecting the $s_{i}$ lines. The second term results in a diagram of the following form after connecting the $l_{i}$ lines:

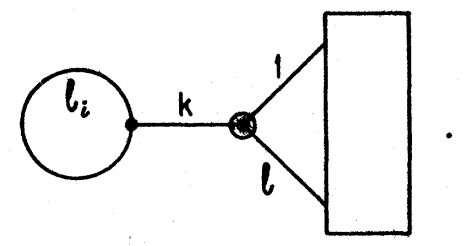

By rule (A4) in Appendix A, $l$ must equal unity. This causes $Q_{l k}$, represented by the circle about the (kl1) vertex, to vanish due to its factor $\left(\begin{array}{ccc}l_{i} & l & l_{i} \\ 0 & 0 & 0\end{array}\right)$, which is zero for odd $l$.

Summarizing (i) and (ii), we have for the direct terms

$$
\begin{aligned}
D(a i b ; c i b) & =\sum_{m_{i} \mu_{i}}\left\langle\phi_{a b}(13) \phi_{i}(2)\left|\left(V_{12}+V_{21}\right)\right| \phi_{c b}^{\prime}(13) \phi_{i}(2)\right\rangle \\
& =\alpha^{2}\left(2 l_{i}+1\right) R_{0}^{(3)}(a i ; c i) \delta_{l_{a} l_{c}} g(a b ; c b),
\end{aligned}
$$

where the Kronecker delta $\delta_{l_{a} l_{c}}$ is due to the factor $\left(\begin{array}{ccc}l_{a} & l & l_{c} \\ 0 & 0 & 0\end{array}\right)$, and $g(a b ; c b)$ is defined by

$$
\begin{aligned}
g(a b ; c b)= & (-)^{J+S+S^{\prime}+l_{a}+l_{b}+1}\left[(2 L+1)\left(2 L^{\prime}+1\right)(2 S+1)\left(2 S^{\prime}+1\right)\right]^{1 / 2} \\
& \times\left(\frac{3}{2}\right)^{1 / 2}\left[\left(2 l_{a}+1\right) l_{a}\left(l_{a}+1\right)\right]^{1 / 2}\left\{\begin{array}{ccc}
S & S^{\prime} & 1 \\
\frac{1}{2} & \frac{1}{2} & \frac{1}{2}
\end{array}\right\}\left\{\begin{array}{lll}
L^{\prime} & L & 1 \\
l_{a} & l_{c} & l_{b}
\end{array}\right\}\left\{\begin{array}{ccc}
S & L & J \\
L^{\prime} & S^{\prime} & 1
\end{array}\right\} .
\end{aligned}
$$

Note that for $a=c, g(a b ; a b)=\left\langle\phi_{a b}(12)\left|\overrightarrow{\mathrm{L}}_{1} \cdot \overrightarrow{\mathrm{s}}_{1}\right| \phi_{a b}^{\prime}(12)\right\rangle$.

(iii) The exchange counterpart of (43) is represented graphically as

$$
\left\langle\phi_{a b}(13) \phi_{i}(2)\left|V_{12}\right| \phi_{c b}^{\prime}(23) \phi_{i}(1)\right\rangle
$$

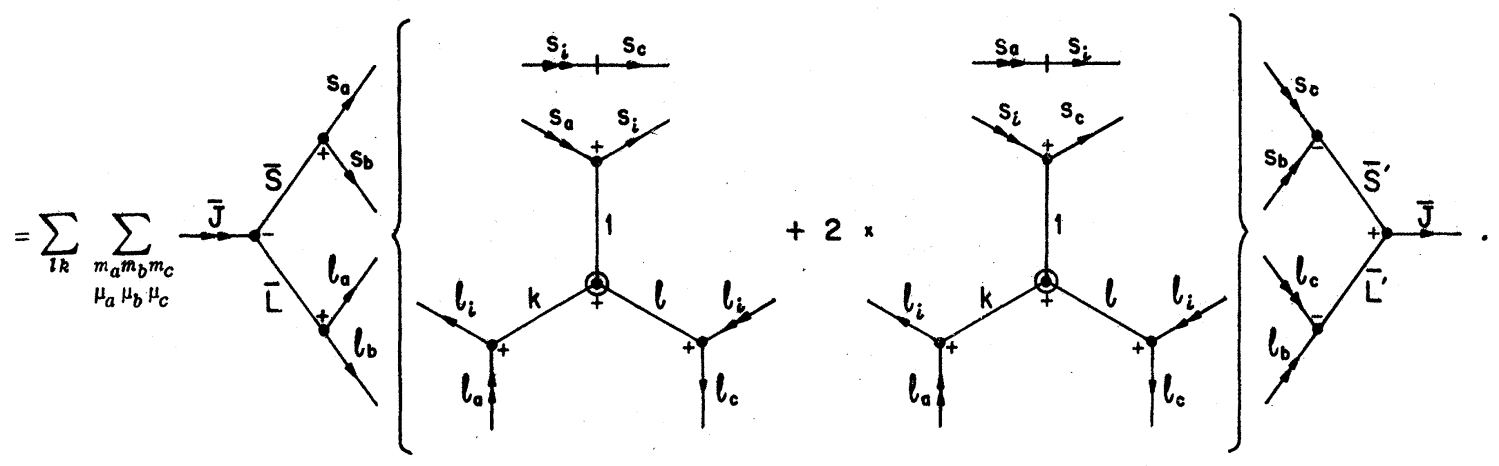

After summing over the closed subshell $i$, the first term of (51) gives the following connected diagram:

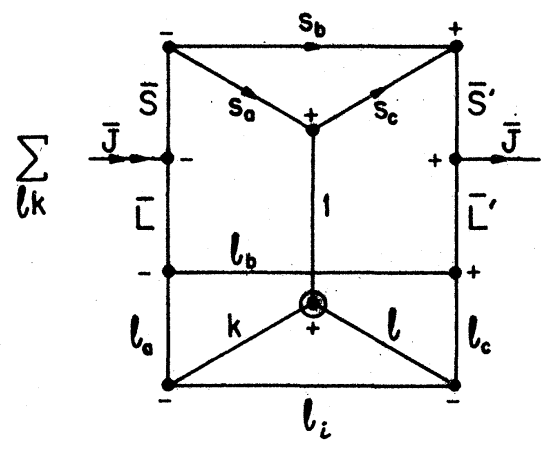


The analytical value of this diagram is

$$
\begin{aligned}
\sum_{l k} Q_{l k}(a i ; i c)(-)^{J+S+S^{\prime}+l_{b}+l_{c}+k+l}\left[(2 L+1)\left(2 L^{\prime}+1\right)(2 S+1)\left(2 S^{\prime}+1\right)\right]^{1 / 2} \\
\quad \times\left\{\begin{array}{lll}
S & S^{\prime} & 1 \\
\frac{1}{2} & \frac{1}{2} & \frac{1}{2}
\end{array}\right\}\left\{\begin{array}{lll}
l & k & 1 \\
l_{a} & l_{c} & l_{i}
\end{array}\right\}\left\{\begin{array}{lll}
S & L & J \\
L^{\prime} & S^{\prime} & 1
\end{array}\right\}\left\{\begin{array}{lll}
L^{\prime} & L & 1 \\
l_{a} & l_{c} & l_{b}
\end{array}\right\} .
\end{aligned}
$$

Other than the factor of 2 , the second term of (51) gives the same connected diagram as (52). Therefore, its contribution is twice the value (53).

(iv) $\left\langle\phi_{a b}(13) \phi_{i}(2)\left|V_{21}\right| \phi_{c b}^{\prime}(23) \phi_{i}(1)\right\rangle$ : Similarly, this exchange counterpart of (ii) can be evaluated, and the total contribution of the corresponding graph is

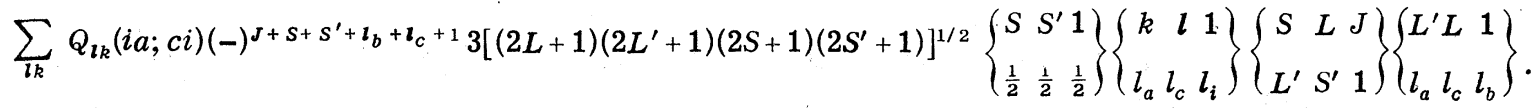

From (iii) and (iv), we obtain the exchange contribution

$$
\begin{aligned}
E(a i b ; i c b) & =\sum_{m_{i} \mu_{i}}\left\langle\phi_{a b}(13) \phi_{i}(2)\left|\left(V_{12}+V_{21}\right)\right| \phi_{c b}^{\prime}(23) \phi_{i}(1)\right\rangle \\
& =6\left(2 l_{i}+1\right)\left(\frac{2 l_{c}+1}{l_{a}\left(l_{a}+1\right)}\right)^{1 / 2} g(a b ; c b) \sum_{l k}\left(\begin{array}{lll}
l_{a} & l & l_{i} \\
0 & 0 & 0
\end{array}\right)\left(\begin{array}{lll}
l_{c} & l & l_{i} \\
0 & 0 & 0
\end{array}\right) T_{l_{k}},
\end{aligned}
$$

where $g(a b ; c b)$ has been defined in $(50)$, and $T_{l k}$ is defined as

$$
\begin{aligned}
T_{l_{k}}= & {[(2 l+1) l(l+1)]^{1 / 2}\left\{\begin{array}{lll}
l & l & 1 \\
l_{a} & l_{c} & l_{i}
\end{array}\right\}\left[R_{l}^{(2)}(a i ; i c)-R_{l}^{(2)}(i a ; c i)\right] \delta_{l_{k}} } \\
& +(-)^{k+l+1}(2 k+1)\left[\left(2 l_{i}+1\right) l_{i}\left(l_{i}+1\right)\right]^{1 / 2}\left\{\begin{array}{lll}
l_{i} & l & l_{a} \\
k & l_{i} & 1
\end{array}\right\}\left\{\begin{array}{lll}
l_{c} & l & l_{i} \\
k & l_{a} & 1
\end{array}\right\} I_{l_{k}}(a i ; i c) \\
& +(2 k+1)\left[\left(2 l_{c}+1\right) l_{c}\left(l_{c}+1\right)\right]^{1 / 2}\left\{\begin{array}{lll}
l_{c} & l & l_{i} \\
k & l_{c} & 1
\end{array}\right\}\left\{\begin{array}{lll}
l_{a} & l & l_{i} \\
k & l_{c} & 1
\end{array}\right\} I_{l_{k}}(i a ; c i) .
\end{aligned}
$$

In terms of (49) and (55), we can express the general matrix element of the mutual spin-orbit interaction between the outer electrons and the closed-shell electrons as

$$
\left\langle\phi_{a b}(12) \phi_{\text {closed }}\left|\sum_{i \neq j}^{\text {(open,closed) }} V_{i j}\right| \phi_{c b}^{\prime}(12) \phi_{\text {closed }}\right\rangle_{A}=\sum_{n_{i} l_{i}}^{\text {closed }}[D(a i b ; c i b)-E(a i b ; i c b)],
$$

where the summation symbol on the left indicates that only open-closed and closed-open pairs are to be included, and the summation on the right is over all closed shells.

\section{Mutual spin-orbit interaction between closed-shell electrons}

The vanishing of this contribution has been noted by Blume and Watson. ${ }^{17}$ This can be seen easily by considering the appropriate $m$-scheme matrix element

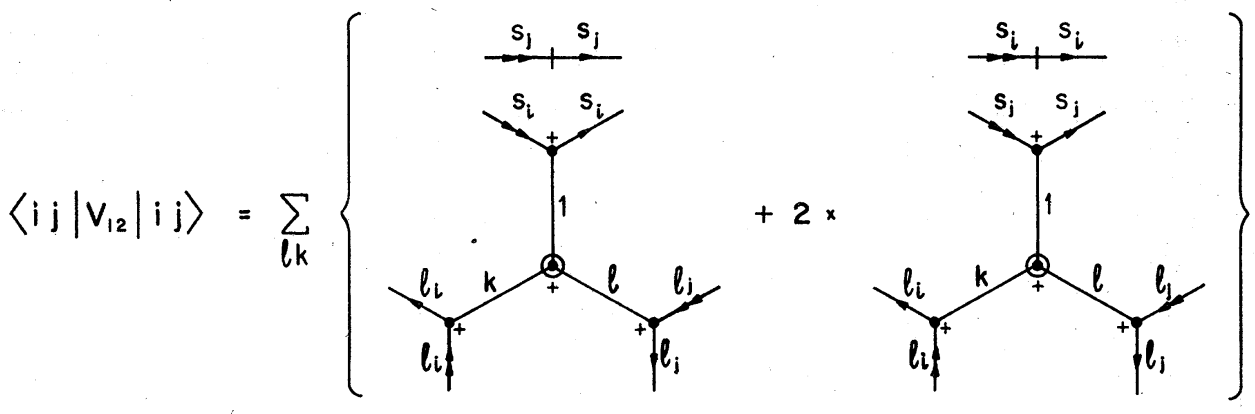


where $i$ and $j$ may or may not represent the same subshell. Upon summing over the closed subshell either $i$ or $j$ [which corresponds to joining the concerned contravariant and covariant lines of (58)], we obtain diagrams having the form of (46) or (48), which give zero contribution. The exchange diagrams of (58) also vanish upon summing over a closed subshell because they lead to diagrams having the form of (46).

\section{Summary remarks}

The graphical technique developed in this section may be used for the calculation of mutual spinorbit matrix elements between arbitrary configurations. The construction of more complicated configuration diagrams may follow El Baz and Castel. ${ }^{26}$ As stated in the beginning of Sec. III, matrix elements of a two-particle operator in the many-particle configuration can always be expressed as linear combinations of matrix elements in corresponding two-particle configurations. Graphically, this corresponds to joining contravariantcovariant angular-momentum pairs in a diagram similar to (37), with appropriate diagrams for the interacting configurations. All the coefficients concerning the coupling scheme of the interacting configurations are contained in the resulting connected diagram, which is similar to (38). Alternatively, we may adopt an analytical approach by using the analytical expression $(25)$ of $G_{l_{k}}(a b ; c d)$ instead of its graphical version (24) although this approach is more laborious than the graphical one.

We summarize in Appendix $C$ the results for the matrix element obtained in this section in the special case of interacting configurations having two electrons outside closed shells. Comparison is also made with certain previously tabulated results in particular cases.

\section{V. $m$-SCHEME MUTUAL SPIN-ORBIT DIAGRAM APPROPRIATE FOR USE WITH BRIGGS' CONFIGURATION DIAGRAMS}

Briggs' paper ${ }^{27}$ on graphical methods of evaluating matrix elements only considered spin-independent interactions in $L S$ coupling; therefore Briggs found it convenient to consider spin and orbital angular-momentum graphs separately. In this section we show first that in LS coupling, even spindependent interaction diagrams can always be factored into spin and orbital parts. We then present our $m$-scheme spin-orbit matrix element (28) in an alternative form appropriate for use with Briggs' configuration diagrams.

For any interacting $L S$-coupled configurations, the matrix element of spin-orbit interaction can always be factored into a product of an orbital diagram, a spin diagram, and a 6-j symbol accounting for the transformation from the $L S M_{S} M_{L}$ scheme to the LSJM scheme. An example is shown by (39). This factorization is due to the fact that spin and orbital momenta are coupled separately for $L S$-coupled configurations. Specifically, in the diagram below we have two arbitrary $L S$-coupled configurations at the top and bottom whose total spin and orbital angular momenta are coupled to total angular momentum $J$. In the middle is the $m$-scheme mutual spin-orbit diagram (28):

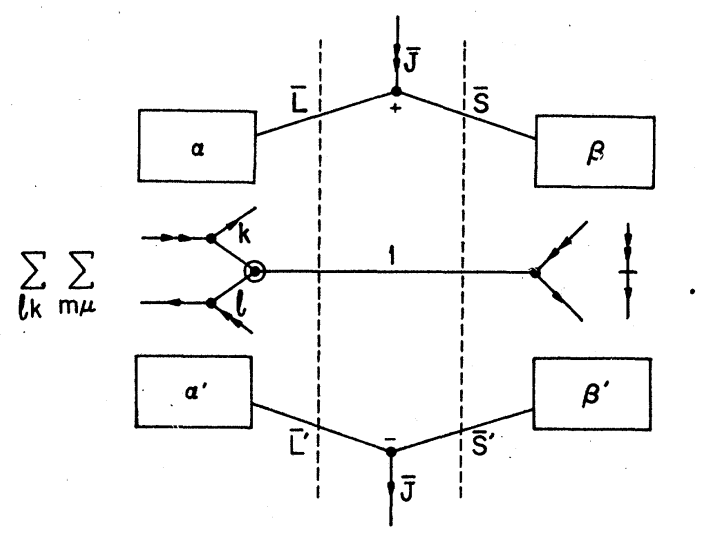

By separating the diagram along the dotted lines according to rule (A4) we obtain the desired factorization:

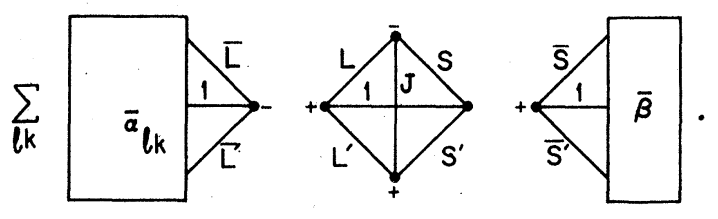

(59)

Here the diagram on the left is an orbital diagram, the diagram on the right is a spin diagram, and the diagram in the middle represents a $6-j$ symbol describing the coupling of total spin and orbital angular momenta.

Alternatively, we can factor the matrix element of mutual spin-orbit interaction in the $m$ scheme into a spin part and an orbital part from the outset, and obtain diagrams in the spin and orbital space separately. Although this procedure does not simplify our calculation in any way, it does give some physical insight. We first factor (25) into a spin part and an orbital part:

$$
\begin{aligned}
G_{l_{k}}(a b ; c d)=\sum_{\nu} & {\left[S_{\nu}(a b ; c d)+2 S_{\nu}(b a ; d c)\right] } \\
& \times L_{l k}^{\nu}(a b ; c d),
\end{aligned}
$$




$$
S_{\nu}(a b ; c d)=\delta_{\mu_{b} \mu_{d}:}\left(\begin{array}{lll}
\mu_{a} & 1 & s_{c} \\
s_{a} & \nu & \mu_{c}
\end{array}\right)
$$

and

$$
L_{l k}^{\nu}(a b ; c d)=\left(\begin{array}{llr}
m_{a} & k & l_{c} \\
l_{a} & m_{a}-m_{c} & m_{c}
\end{array}\right)\left(\begin{array}{lrr}
m_{b} & l & l_{d} \\
l_{b} & m_{b}-m_{d} & m_{d}
\end{array}\right)\left(\begin{array}{ccc}
m_{a}-m_{c} & m_{b}-m_{d} \nu \\
k & l & 1
\end{array}\right) .
$$

Note that the summation over the magnetic quantum number $\nu$ only exists formally. Thus we can rewrite (23) as

$$
\left\langle a b\left|V_{12}\right| c d\right\rangle=\sum_{\nu} \mathcal{S}_{\nu} \mathcal{L}^{\nu}
$$

where the spin part is denoted by

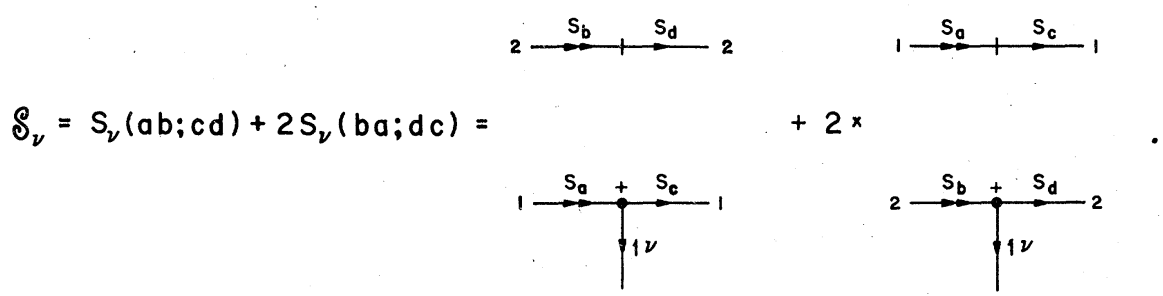

Note that, when taking the matrix element in the spin space, the free angular-momentum line $1 \nu$ in $(64)$ is to be joined with the total spins of the two interacting configurations to form a closed diagram multiplied by a diagram representing a 3-j symbol [cf. rule (A4)]. The orbital part of (63) is denoted by

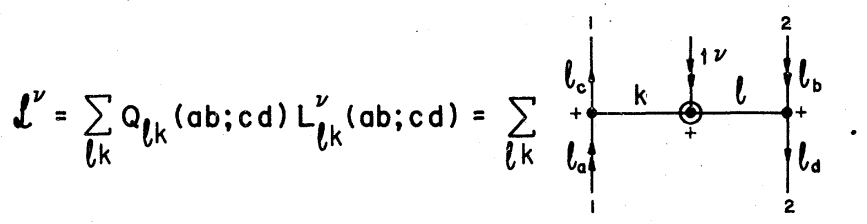

Similarly, when taking the matrix element in the orbital space, the free angular-momentum line $1 \nu$ is to be joined with the total orbital angular momenta of the two interacting configurations. The two 3-j diagrams obtained, one from the spin space and the other from the orbital space, will be combined with two other $3-j$ diagrams, accounting for the coupling of the total spin and orbital angular momenta to the total $J$ in the two interacting configurations, to form a $6-j$ diagram. The final result has been shown schematically by (59).

Upon changing the signs of the nodes in (64) and (65) and eliminating the double arrow notation, we obtain mutual spin-orbit diagrams in the $m$ scheme suitable for use with Briggs '27 LS-coupled configuration diagrams. Thus we represent (64) as

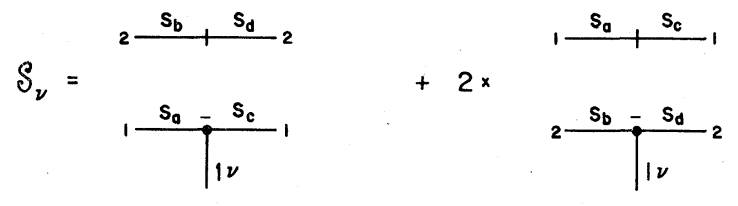

(66)

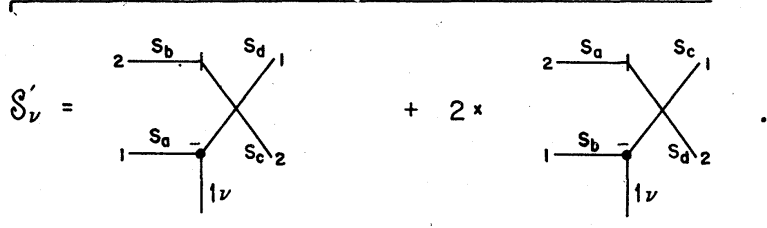

Similarly, we can represent (65) as<smiles>CCC(C)C(CC)C(C)(C)C(C)(C)C</smiles>

and its exchange counterpart as

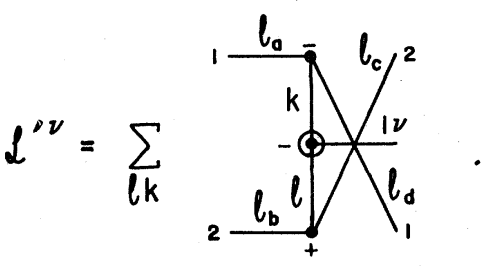


Here the circle at the vertex involving $k$ and $l$ represents the multiplication factor $Q_{l k}(a b ; c d)$ in Eq. (26). When using the diagrams in (66)-(69) in conjunction with Briggs' rules $^{27}$ for computing matrix elements, Fig. (3.11) of Ref. 27 should be consulted. Firstly, Briggs' $\rho, \sigma, \rho^{\prime}, \sigma^{\prime}$ notations correspond to our $a, b, c, d$. Secondly, our diagrams (66) and (68) correspond to the spin and orbital parts of Briggs' general diagram (3.11a); our (67) and (69) to his (3.11b); our (66) and (68) in which lines $a$ and $b$ are crossed to Briggs' (3.11c); and lastly, our (67) and (69) in which lines $a$ and $b$ are crossed to his (3.11d). The matrix element of the spin-orbit interaction between arbitrary configurations is now obtained by evaluating the matrix element of each of the four spin and orbital interaction diagrams corresponding to $(3.11 \mathrm{a}-\mathrm{d})$ in Ref. 27 using the rules in Sec. 6.1 of Ref. 27. When applying these rules the following minor modifications of rules (iii) and (x) should be observed:

(iii) There is an overall factor (-1) corresponding to Briggs' diagram (3.11b) and (3.11c); $(x)$ When connecting the free $1 \nu$ line in (66)-(69) with total spin and orbital angular momentum, the sign conventions in our diagram (59) should be followed. Lastly, the $6 j$ symbol in our (59),

$$
(-1)^{L+S+J+L+L^{\prime}+1}\left\{\begin{array}{lll}
L & L^{\prime} & 1 \\
S^{\prime} & S & J
\end{array}\right\},
$$

multiplies the overall matrix element.

\section{SPIN-ORBIT INTERACTION IN A CENTRAL FIELD}

For completeness, we present here a graphical treatment for the trivial case of spin-orbit interaction in a central field. The interaction Hamiltonian assumes the general form

$$
V_{s o}^{(c)}=\sum_{i=1}^{N} \zeta\left(r_{i}\right) \overrightarrow{\mathrm{L}}_{i} \cdot \overrightarrow{\mathrm{s}}_{i} .
$$

Any two interacting configurations can differ at most by one orbital, although they must have the same set of angular-momentum quantum numbers. We first derive the matrix element in the $m$ scheme,

$$
\begin{aligned}
\left\langle\phi_{n_{a} l^{l} m_{a} \mu_{a}}(i)\left|\zeta\left(r_{i}\right) \overrightarrow{\mathrm{L}}_{i} \cdot \overrightarrow{\mathrm{s}}_{i}\right| \phi_{n_{e^{l}} c^{m} c^{\mu} c}(i)\right\rangle \\
=\zeta_{a c}\left(a\left|\overrightarrow{\mathrm{L}}_{i} \cdot \overrightarrow{\mathrm{s}}_{i}\right| c\right),
\end{aligned}
$$

where the radial integral $\zeta_{a c}$ is defined by

$$
\zeta_{a c}=\int_{0}^{\infty} r_{i}^{2} d r_{i} R_{n_{a} l_{a}}\left(r_{i}\right) \zeta\left(r_{i}\right) R_{n_{c} l_{c}}\left(r_{i}\right)
$$

and we use the rounded bra $(a \mid$ and ket $\mid c)$ to denote the angular and spin part of the $m$-scheme states $\left\langle\phi_{n_{a} l_{a} m_{a} \mu_{a}}(i)\right|$ and $\left|\phi_{n_{c} l_{c} m_{c} \mu_{c}}(i)\right\rangle$, respectively. Making use of (9) and (10), we can easily obtain

$$
\left(a\left|\overrightarrow{\mathrm{L}}_{i} \cdot \overrightarrow{\mathrm{s}}_{i}\right| c\right)=\left(\frac{3}{2}\right)^{1 / 2}\left[\left(2 l_{a}+1\right) l_{a}\left(l_{a}+1\right)\right]^{1 / 2} G(a ; c),
$$

where

$$
G(a ; c)=\delta_{l_{a} l_{c}} \sum_{\nu}\left(\begin{array}{lll}
m_{a} & l_{c} & \nu \\
l_{a} & m_{c} & 1
\end{array}\right)\left(\begin{array}{ccc}
\mu_{a} & 1 & \frac{1}{2} \\
\frac{1}{2} & \nu & \mu_{c}
\end{array}\right),
$$

or, graphically,

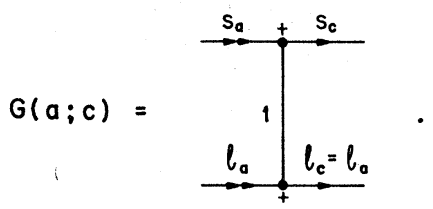

Here the summation over $\nu$ only exists formally since $\nu=m_{c}-m_{a}=\mu_{c}-\mu_{a}$ due to the conservation of the "magnetic current" at each node or, analytically, due to the selection rule for magnetic quantum numbers of the covariant $3-j$ symbol. Thus we can write (71) as

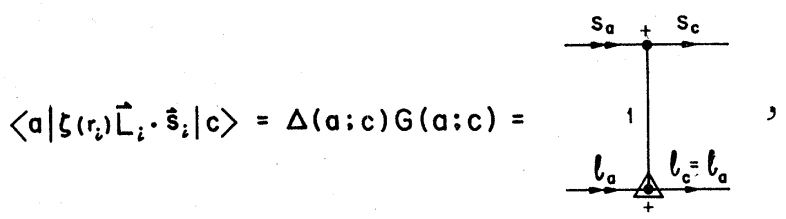

where $\Delta(a ; c)$ is defined as

$$
\Delta(a ; c)=\zeta_{a c}\left(\frac{3}{2}\right)^{1 / 2}\left[\left(2 l_{a}+1\right) l_{a}\left(l_{a}+1\right)\right]^{1 / 2},
$$

and represented by a triangle at the node involving $l_{a}$ and $l_{c}$.

As an example of the use of (76) to compute matrix elements of (70) between $L S$-coupled states, we will once again treat the case of configurations having two nonequivalent electrons outside any number of closed shells. The matrix element of spin-orbit interaction of an electron in the closed subshell $j$,

$$
\left\langle\phi_{n_{j} l_{j} m_{j} \mu_{j}}(i)\left|\zeta\left(r_{i}\right) \overrightarrow{\mathrm{L}}_{i} \cdot \overrightarrow{\mathrm{s}}_{i}\right| \phi_{n_{j} l_{j} m_{j} \mu_{j}}(i)\right\rangle,
$$

can be represented graphically as

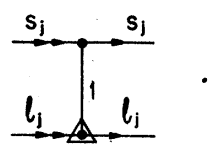

The summation over the magnetic quantum number $m_{j}$ or $\mu_{j}$ corresponds to joining the free ends of angular-momentum lines $l_{j}$ or $s_{j}$, respectively. This gives zero contribution, as may be seen immediately by virtue of (46). Hence the summation over electrons in closed shells vanishes. 
For the spin-orbit interaction of the outer electrons, the two interacting configurations may differ by one orbital. Therefore, we consider the matrix element $\left\langle\phi_{a b}(12)\left|\zeta\left(r_{1}\right) \overrightarrow{\mathrm{L}}_{1} \cdot \overrightarrow{\mathrm{s}}_{1}\right| \phi_{c b}^{\prime}(12)\right\rangle$, which has the graphical form

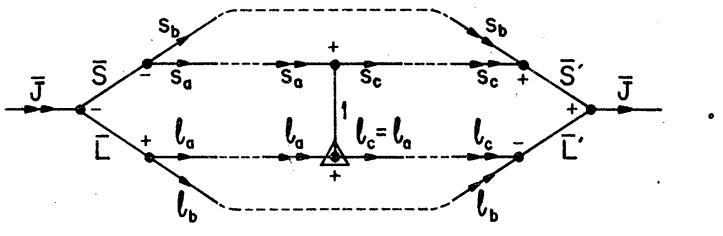

Its analytical value is

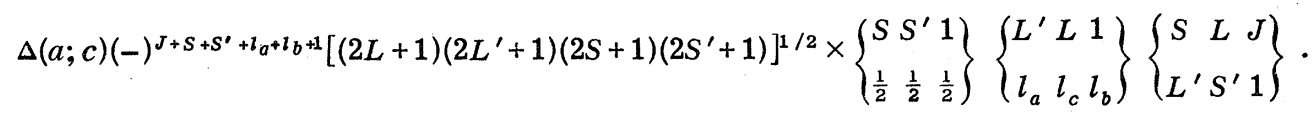

Thus the general matrix element for the whole atom is given by

$$
\left\langle\phi_{a b}(12) \phi_{c \text { losed }}\left|\sum_{i=1}^{N} \zeta\left(r_{i}\right) \overrightarrow{\mathrm{L}}_{i} \cdot \overrightarrow{\mathrm{s}}_{i}\right| \phi_{c b}^{\prime}(12) \phi_{c 10 s e d}\right\rangle_{A}=\left\langle\phi_{a b}(12)\left|\zeta\left(r_{1}\right) \overrightarrow{\mathrm{L}}_{1} \cdot \overrightarrow{\mathrm{s}}_{1}\right| \phi_{c b}^{\prime}(12)\right\rangle=\delta_{l_{a} l_{c}} \zeta_{a c} g(a b ; c b),
$$

where $g(a b ; c b)$ and $\zeta_{a c}$ have been defined in $(50)$ and (72), respectively. 'The extension of the present graphical method to more complex configurations is straightforward and can easily be obtained by using (76) and graphical representations of the corresponding configurations. ${ }^{26,27}$ There is also an existing computer program by $\mathrm{Klotz}^{32}$ to compute matrix elements of (70) using an analytical approach.

\section{DISCUSSION}

A. Analysis of the various contributions to the mutual spin-orbit interaction

In order to review the physical meaning of the mutual spin-orbit interaction, we write the mutual spin-orbit interaction between electrons $i$ and $j$ in the form ${ }^{2}$

$$
\begin{aligned}
V(i j)= & -\alpha^{2}\left\{\frac{\left(\overrightarrow{\mathrm{r}}_{i}-\overrightarrow{\mathrm{r}}_{j}\right)}{r_{i j}^{3}} \times\left(\overrightarrow{\mathrm{p}}_{i}-\overrightarrow{\mathrm{p}}_{j}\right)-\frac{\left(\overrightarrow{\mathrm{r}}_{i}-\overrightarrow{\mathrm{r}}_{j}\right)}{2 r_{i j}^{3}} \times \overrightarrow{\mathrm{p}}_{i}\right\} \cdot \overrightarrow{\mathrm{s}}_{i} \\
& -\alpha^{2}\left\{\frac{\left(\overrightarrow{\mathrm{r}}_{j}-\overrightarrow{\mathrm{r}}_{i}\right)}{r_{i j}^{3}} \times\left(\overrightarrow{\mathrm{p}}_{j}-\overrightarrow{\mathrm{p}}_{i}\right)-\frac{\left(\overrightarrow{\mathrm{r}}_{j}-\overrightarrow{\mathrm{r}}_{i}\right)}{2 r_{i j}^{3}} \times \overrightarrow{\mathrm{p}}_{j}\right\} \cdot \overrightarrow{\mathrm{s}}_{j} .
\end{aligned}
$$

Here inside the first pair of curly brackets, the first term may be derived from classical arguments. It is proportional to the angular momentum of the $i$ th electron with respect to the $j$ th electron. The second term is of purely kinematic origin due to a relativistic effect called the Thomas precession. ${ }^{33}$ We have a similar account for the terms in the second pair of curly brackets. Physically, the expression in the first pair of curly brackets is proportional to the effective magnetic field experienced by the $i$ th electron with respect to the $j$ th electron, and the expression in the second pair of curly brackets is proportional to the effective magnetic field experienced by the $j$ th electron with respect to the $i$ th electron. Since the spin angularmomentum operator is proportional to the spin magnetic moment of the electron, the mutual spinorbit interaction (81) simply represents a certain type of mutual magnetic interaction between electrons. On grouping separately terms involving $\overrightarrow{\mathrm{p}}_{i}$ and $\overrightarrow{\mathrm{p}}_{j}$, we retrieve the commonly used form

$$
\begin{aligned}
V(i j)= & -\frac{\alpha^{2}}{2}\left\{\frac{\left(\overrightarrow{\mathrm{r}}_{i}-\overrightarrow{\mathrm{r}}_{j}\right)}{r_{i j}^{3}} \times \overrightarrow{\mathrm{p}}_{i}\right\} \cdot\left(\overrightarrow{\mathrm{s}}_{i}+2 \overrightarrow{\mathrm{s}}_{j}\right) \\
& -\frac{\alpha^{2}}{2}\left\{\frac{\left(\overrightarrow{\mathrm{r}}_{j}-\overrightarrow{\mathrm{r}}_{i}\right)}{r_{i j}^{3}} \times \overrightarrow{\mathrm{p}}_{j}\right\} \cdot\left(\overrightarrow{\mathrm{s}}_{j}+2 \overrightarrow{\mathrm{s}}_{i}\right) .
\end{aligned}
$$

In this discussion, we shall call terms involving $\overrightarrow{\mathrm{p}}$ and $\overrightarrow{\mathrm{s}}$ of the same electron the spin-self-orbit interaction, and terms involving $\vec{p}$ and $\vec{s}$ of different electrons the spin-other-orbit interaction. The mutual spin-orbit interaction has been and will be used to refer to both the spin-self-orbit and spinother-orbit interactions. To facilitate our discussion, we shall further call those terms included in the first pair of curly brackets in (81) the mutual spin-orbit interaction in the field of the $j$ th electron, and those terms included in the second pair of curly brackets in (81) the mutual spin-orbit interaction in the field of the $i$ th electron. In the zero-order coupling scheme, each contribution to the total mutual spin-orbit interaction between two electrons can be summarized using the graphical diagram (24):

(i) Spin-self-orbit interaction of the electron 1 in the field of the electron 2: 


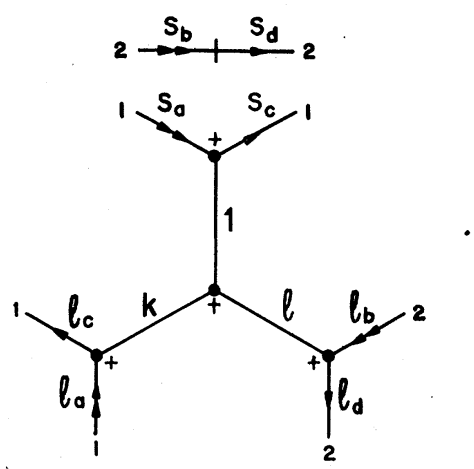

(ii) Spin-other-orbit interaction of the electron 1 in the field of the electron 2 :

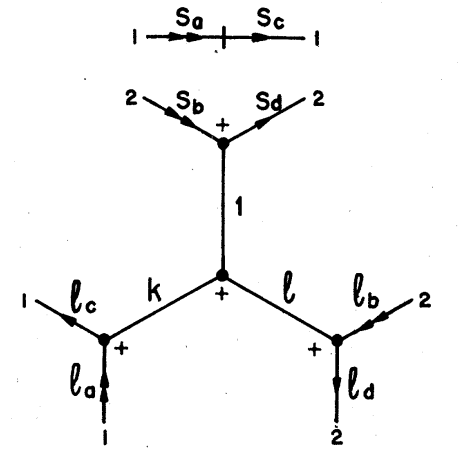

(iii) Spin-self-orbit interaction of the electron 2 in the field of the electron 1 :

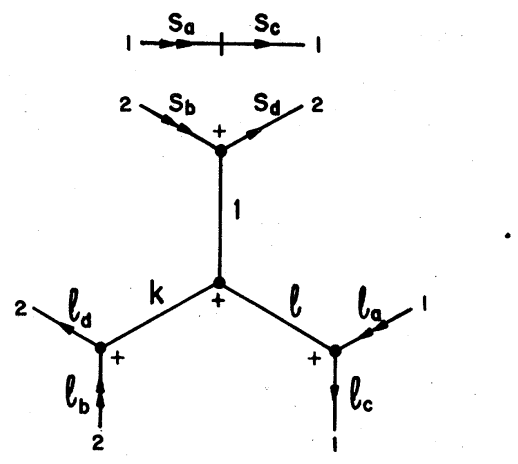

(iv) Spin-other-orbit interaction of the electron 2 in the field of the electron 1 :

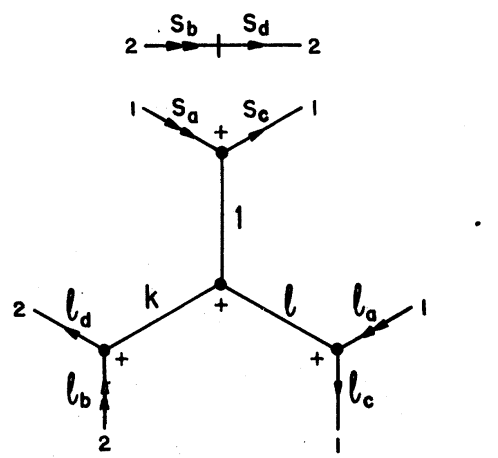

B. Graphical reduction of the two-particle spin-orbit interaction to an effective one-particle spin-orbit interaction in certain cases

There are certain simplifications when considering the mutual spin-orbit interaction between an open-shell electron and a closed shell within the Hartree-Fock description of the atom.

(i) 'The direct contribution to the spin-self-orbit interaction of an open-shell electron in the field of a closed shell always behaves like an effective one-particle spin-orbit interaction. This can be seen by considering the corresponding diagram

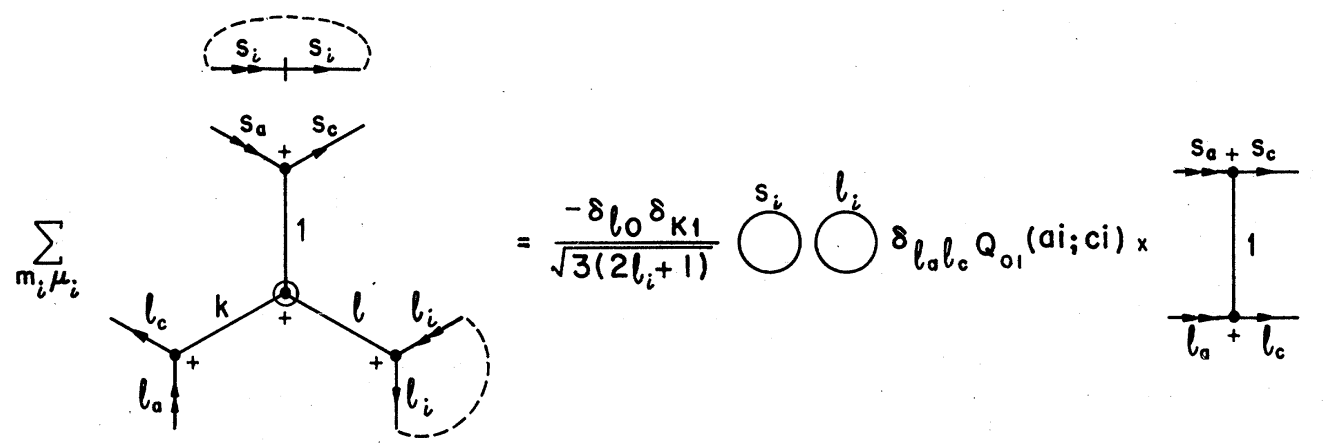

which couples (or connects graphically) the interacting configurations in the same way as the one-particle spin-orbit interaction (76). Here the Kronecker delta $\delta_{l_{a}} l_{c}$ is due to the factor $\left(\begin{array}{lll}l_{a} & l & l_{c} \\ 0 & 0 & 0\end{array}\right)$ in $Q_{l_{k}}$, which is denoted by the circle about the $(k l 1)$ node. This result can also be deduced by noting that the field of a closed shell is a central one. A specific example for two nonequivalent electrons outside any number of closed shells is illustrated by (44).

(ii) The exchange contribution to the spin-self-orbit interaction of an open-shell electron in the field of a closed shell behaves like an effective one-particle spin-orbit interaction only within a configuration. 
The exchange contribution is

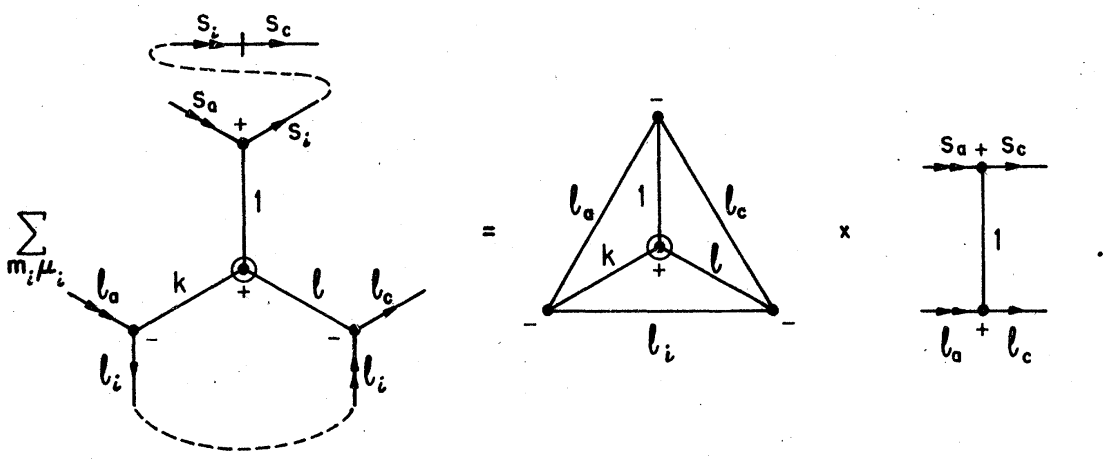

This is an effective one-particle operator. But since we do not have the kronecker delta $\delta_{l_{a} l_{c}}$ here, this only behaves like a one-particle spin-orbit interaction within a configuration, in which case the condition $l_{a}=l_{c}$ is guaranteed.

(iii) The direct contribution to the spin-self-orbit interaction of a closed shell in the field of any other electron vanishes. This can be seen immediately from the diagram

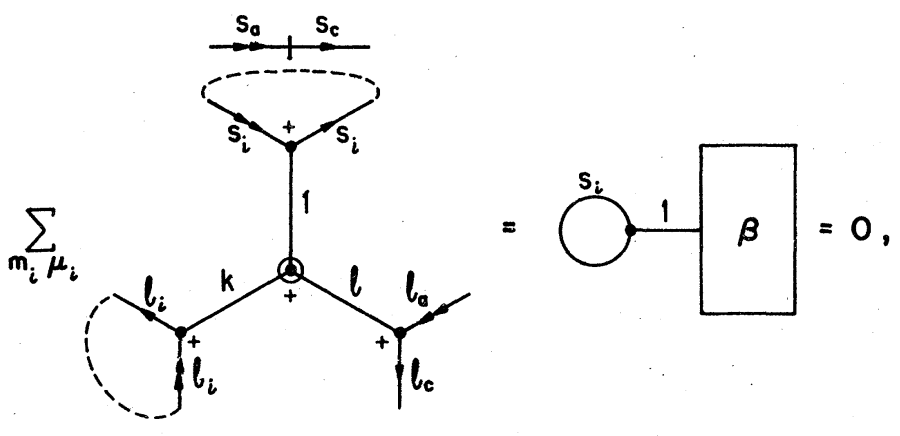

which gives zero contribution due to (46). This may also be expected intuitively by noting that the total spin and orbital angular momenta are zero for a closed shell.

(iv) The exchange contribution to the spin-self-orbit interaction of a closed shell in the field of any other electron is an effective one-particle spin-orbit interaction only within a configuration. The exchange contribution has the diagram
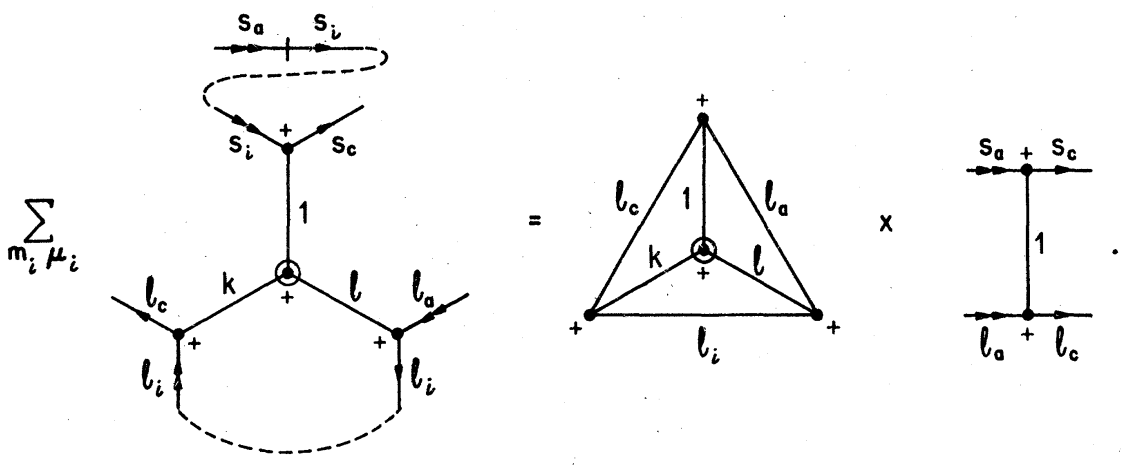

As before, this is a one-particle operator, but only within a configuration can this be treated as an effective one-particle spin-orbit interaction.

(v) For the spin-self-orbit interaction between open-shell electrons, neither the direct nor the exchange contributions can in general be considered as an effective one-particle spin-orbit interaction. Only within the same configuration are part of the contributions proportional to a one-particle spin-orbit interaction, e.g., for $l=0$, 


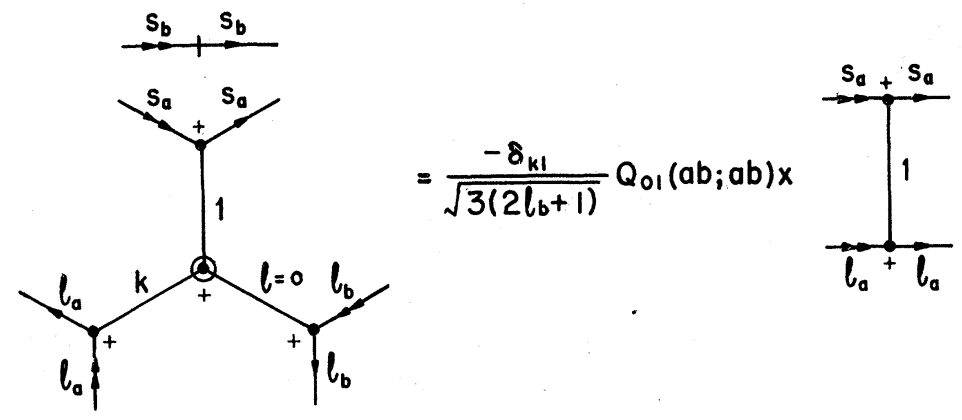

and

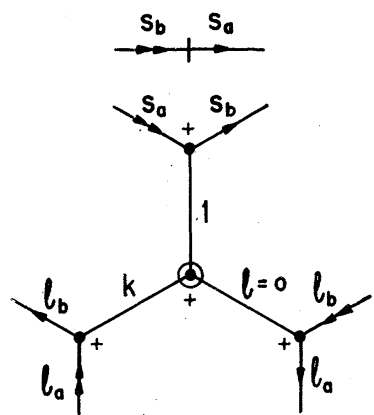

$=\frac{-\delta l_{a} l_{b} \delta_{k 1}}{\sqrt{3\left(2 l_{a}+1\right)}} Q_{01}(a b ; b a) x$

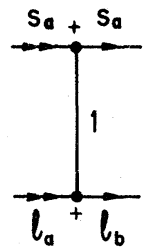

(vi) The direct contribution to the spin-other-orbit interaction of an electron in the field of a closed shell vanishes. Graphically, this is elucidated by the diagram

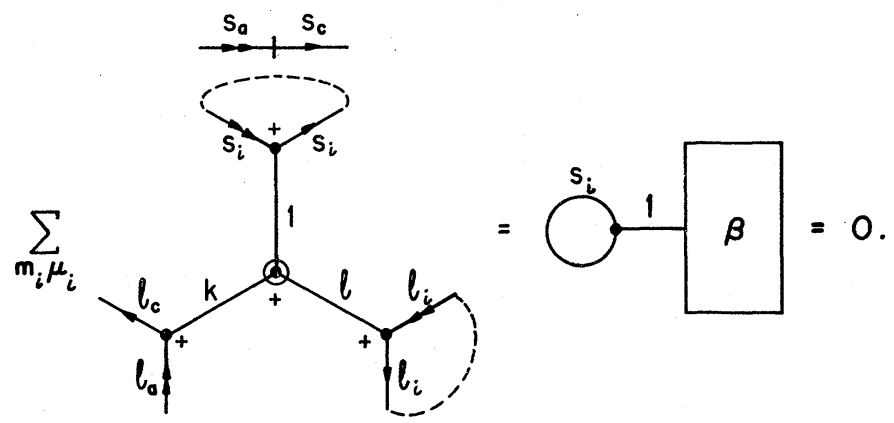

This is what would be expected intuitively since the total orbital angular momentum of a closed shell is zero.

(vii) The exchange contribution to the spin-other-orbit interaction of an electron in the field of a closed shell behaves like an effective one-particle spin-orbit interaction. The diagram for the exchange contribution is the same as its spin-self-orbit counterpart (88), but twice as large.

(viii) The direct contribution to the spin-other-orbit interaction of a closed shell in the field of any other electron vanishes. Graphically, we have

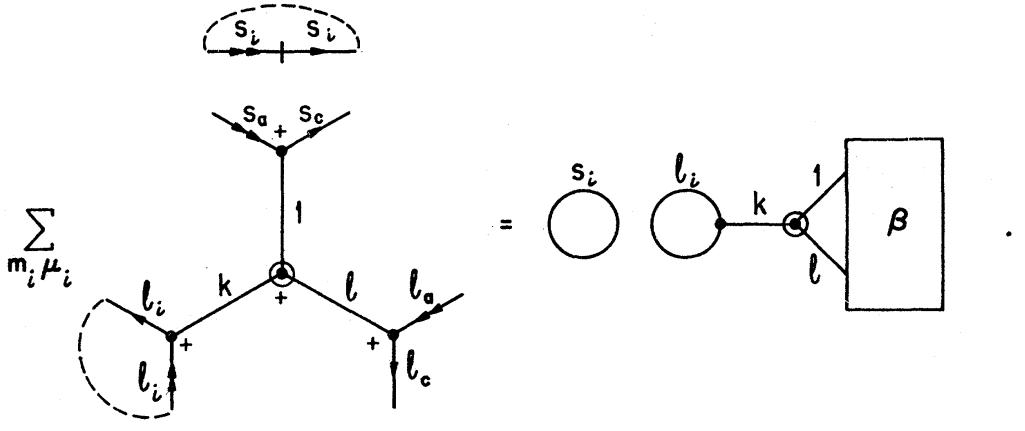


This diagram vanishes for the same reason that diagram (48) vanishés. This conclusion also follows from the intuitive argument that the total spin angular momentum of a closed shell is zero.

(ix) The exchange contribution to the spin-other-orbit interaction of a closed shell in the field of any other electron is an effective one-particle spin-orbit interaction only within a configuration. The diagram for the exchange contribution is the same as its spin-self-orbit counterpart (90), but twice as large.

(x) Only part of the spin-other-orbit interaction between open-shell electrons can be related to an effective one-particle spin-orbit interaction. This result is similar to that for the spin-self-orbit case. For two open-shell electrons (either equivalent or nonequivalent) which are directly coupled to each other, all contributions to the spin-other-orbit interaction between them have a magnitude twice as large as those of their spin-self-orbit counterparts and a relative phase factor $(-)^{s+s^{\prime}}$, where $s$ and $s^{\prime}$ are the resultant spin angular momenta of the electron pair under consideration in the two interacting configurations. We can easily verify this graphically,
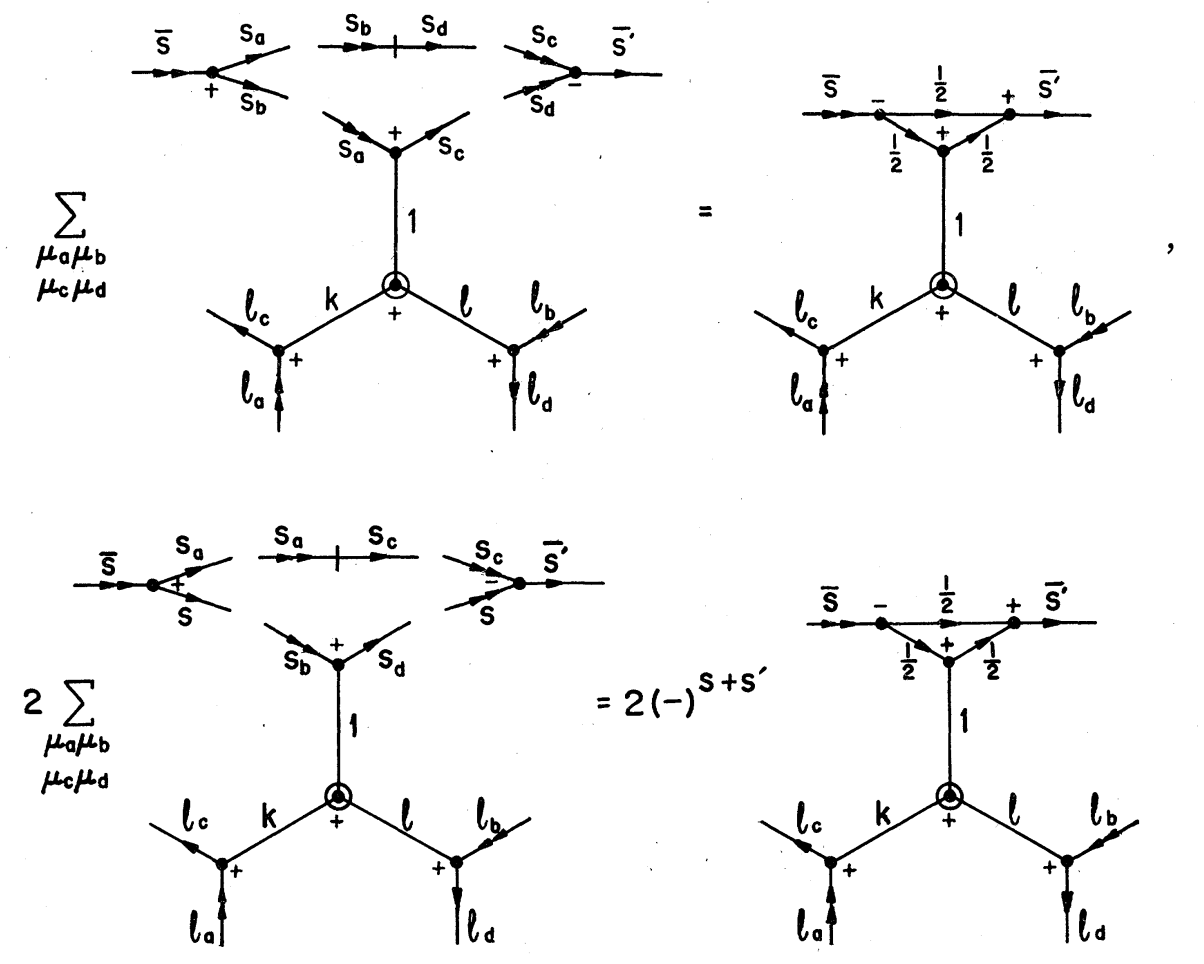

Other cases can be analyzed similarly. However, the relative magnitude and phase factor depend in general on the coupling scheme of the interacting configurations.

Note that except for (95) and (96), the results we have obtained so far were derived without making any assumption about the coupling scheme of the interacting configurations; therefore, they are valid in any coupling scheme. Also note the interesting fact that the spin-other-orbit interaction in many cases contributes twice as much as the spinself-orbit interaction, a fact also noted by Blume and Watson. ${ }^{17}$ This can be attributed to the Thomas precession, ${ }^{33}$ which reduces the spin-self-orbit interaction by half but does not affect the spinother-orbit interaction.

C. Derivation of an effective spin-orbit parameter $\zeta_{c}$ equivalent to that of Blume and Watson

The result that a large part of the mutual spinorbit interaction within the same configuration can be regarded as an effective one-particle spinorbit interaction has been noted implicitly or explicitly by other authors. ${ }^{11-17}$ In fact, a major effort in the past has been the derivation of effective spin-orbit parameters for many-electron atoms. Blume and Watson ${ }^{17}$ derived an effective spin-orbit parameter $\zeta_{c}$ for configurations having a single open shell by considering only the mutual spinorbit interaction between the open- and closedshell electrons. Using our graphical approach, we derive an effective spin-orbit parameter $\zeta_{c}$ 
which is equivalent to that of Blume and Watson. ${ }^{17}$

Blume and Watson's definition for $\zeta_{c}$ can be written

$$
\begin{aligned}
\zeta_{c}\left\langle a\left|\overrightarrow{\mathrm{L}}_{1} \cdot \overrightarrow{\mathrm{s}}_{1}\right| a\right\rangle= & \left\langle a\left|\frac{Z \alpha^{2}}{2} \frac{1}{r_{1}^{3}} \overrightarrow{\mathrm{L}}_{1} \cdot \overrightarrow{\mathrm{s}}_{1}\right| a\right\rangle \\
+ & \sum_{n_{b} l_{b}}^{\text {closed }} \sum_{m_{b} \mu_{b}}\left[\left\langle a b\left|\left(V_{12}+V_{21}\right)\right| a b\right\rangle\right. \\
& \left.-\left\langle a b\left|\left(V_{12}+V_{21}\right)\right| b a\right\rangle\right] .
\end{aligned}
$$$$
\begin{aligned}
\left(a\left|\overrightarrow{\mathrm{L}}_{1} \cdot \overrightarrow{\mathrm{s}}_{1}\right| a\right)= & \left(\frac{3}{2}\right)^{1 / 2}\left[\left(2 l_{a}+1\right) l_{a}\left(l_{a}+1\right)\right]^{1 / 2} \\
& \times \sum_{\nu}\left(\begin{array}{ccc}
m_{a} & l_{a} & \nu \\
l_{a} & m_{a} & 1
\end{array}\right)\left(\begin{array}{ccc}
\mu_{a} & 1 & s_{a} \\
s_{a} & \nu & \mu_{a}
\end{array}\right) \\
= & {\left[\frac{3}{2}\left(2 l_{a}+1\right) l_{a}\left(l_{a}+1\right)\right]^{1 / 2} \times \overbrace{1}^{l_{l_{a}}} . }
\end{aligned}
$$

Here the summation over $\nu$ only exists formally since $\nu=0$.

The second term on the right-hand side of (97) has been calculated more generally in (87)-(90), (93), and (94). By using the analytic expressions associated with those diagrams and substituting the following relations between our radial integrals (21) and the radial integrals $N^{l}(a b), N^{l-2}(a b)$, $V^{l-1}(a b)$, and $M^{0}(a b)$ of Blume and Watson, ${ }^{17}$

$$
\begin{aligned}
R_{l}^{(1)}(a b ; b a) & =R_{l}^{(1)}(b a ; a b)=N^{l-2}(a b)+N^{l}(a b), \\
R_{l}^{(2)}(a b ; b a) & -R_{l}^{(2)}(b a ; a b)=V^{l-1}(a b) \\
R_{0}^{(3)}(a b ; a b) & =-M^{0}(a b) \\
R_{l}^{(3)}(a b ; b a) & =R_{l}^{(3)}(b a ; a b) \\
& =l N^{l-2}(a b)-(l+1) N^{l}(a b),
\end{aligned}
$$

we obtain the following result:

$$
\begin{aligned}
\sum_{n b \iota_{b}}^{\text {closed }} \sum_{m_{b} \mu_{b}}[\langle a b| & \left.\left(V_{12}+V_{21}\right)|a b\rangle-\left\langle a b\left|\left(V_{12}+V_{21}\right)\right| b a\right\rangle\right] \\
& =\left(a\left|\overrightarrow{\mathrm{L}}_{1} \cdot \overrightarrow{\mathrm{s}}_{1}\right| a\right) \sum_{n_{b} l_{b}}^{\text {closed }}\left[-4\left(2 l_{b}+1\right) M^{0}(a b)-6\left(2 l_{b}+1\right)\left(\frac{2 l_{a}+1}{l_{a}\left(l_{a}+1\right)}\right)^{1 / 2} \sum_{l_{k}}\left(\begin{array}{ccc}
l_{a} & l & l_{b} \\
0 & 0 & 0
\end{array}\right)^{2} T_{l_{k}}\right] .
\end{aligned}
$$

Here we have defined

$$
\begin{aligned}
& T_{l k}=[(2 l+1) l(l+1)]^{1 / 2}\left\{\begin{array}{ccc}
l & l & 1 \\
l_{a} & l_{a} & l_{b}
\end{array}\right\} V^{l-1}(a b) \delta_{l_{k}}+t_{l_{k}}(2 k+1)\left\{\begin{array}{lll}
l_{a} & l & l_{b} \\
k & l_{a} & 1
\end{array}\right\} \\
& \times\left[(-)^{k+l+1}\left[\left(2 l_{b}+1\right) l_{b}\left(l_{b}+1\right)\right]^{1 / 2}\left\{\begin{array}{lll}
l_{b} & l & l_{a} \\
k & l_{b} & 1
\end{array}\right\}+\left[\left(2 l_{a}+1\right) l_{a}\left(l_{a}+1\right)\right]^{1 / 2}\left\{\begin{array}{lll}
l_{a} & l & l_{b} \\
k & l_{a} & 1
\end{array}\right\}\right],
\end{aligned}
$$

and

$$
t_{l(l-1)}=(2 l+1) N^{l-2}(a b), \quad t_{l l}=(l+1) N^{l-2}(a b)-l N^{l}(a b), \quad t_{l(l+1)}=-(2 l+1) N^{l}(a b) .
$$

Hence from (97)-(99) and (102), we can write our expression for the spin-orbit parameter as

$$
\zeta_{c}=\frac{Z \alpha^{2}}{2}\left\langle\frac{1}{r_{1}^{3}}\right\rangle_{a}-\sum_{n_{b} l_{b}}^{\text {closed }} 2\left(2 l_{b}+1\right)\left[2 M^{0}(a b)+3\left(\frac{2 l_{a}+1}{l_{a}\left(l_{a}+1\right)^{2}}\right)^{1 / 2} \sum_{l_{k}}\left(\begin{array}{ccc}
l_{a} & l & l_{b} \\
0 & 0 & 0
\end{array}\right)^{2} T_{l k}\right] \text {. }
$$

Alternatively, we can also start from (22a), (22b), and (22c) and by a similar procedure get

$$
\zeta_{c}=\frac{Z \alpha^{2}}{2}\left\langle\frac{1}{r_{1}^{3}}\right\rangle_{a}-\sum_{n_{b} l_{b}}^{\text {closed }} 2\left(2 l_{b}+1\right)\left[2 M^{0}(a b)+3 \sum_{l}\left(\begin{array}{lll}
l_{a} & l & l_{b} \\
0 & 0 & 0
\end{array}\right)^{2} S_{l}\right] \text {, }
$$


where

$$
\begin{aligned}
S_{l}= & \left(\frac{2 l_{a}+1}{l_{a}\left(l_{a}+1\right)}\right)^{1 / 2}[(2 l+1) l(l+1)]^{1 / 2}\left\{\begin{array}{ccc}
l_{a} & l_{a} & 1 \\
l & l & l_{b}
\end{array}\right\}\left[N^{l}(a b)+N^{l-2}(a b)-V^{l-1}(a b)\right] \\
& +\left[-1+\left(\frac{2 l_{a}+1}{l_{a}\left(l_{a}+1\right)}\right)^{1 / 2}\left[\left(2 l_{b}+1\right) l_{b}\left(l_{b}+1\right)\right]^{1 / 2}\left\{\begin{array}{ll}
l_{a} l_{a} & 1 \\
l_{b} l_{b} & l
\end{array}\right\}\right]\left[l^{l-2}(a b)-(l+1) N^{l}(a b)\right] .
\end{aligned}
$$

The expressions (105) and (106) and Blume and Watson's ${ }^{17}$ expression for the spin-orbit parameter $\zeta_{c}$ are all equivalent to each other. Although a general proof of equivalence is not given here, one can easily show the equivalence for the specific cases $l_{b}=0, l_{b}=1$, etc. by substituting the corresponding values for $l_{b}$ in the expressions.

\section{APPENDIX A: TRANSFORMATION RULES FOR GRAPHS}

There are only two fundamental transformation rules: Rule (1):

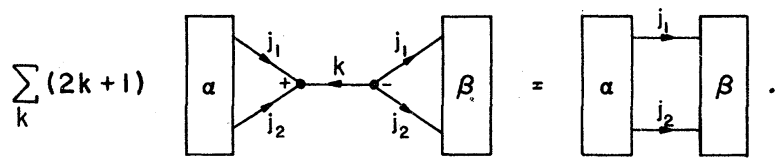

(A1)

This rule follows from the graphical relation for $3-j$ symbols,

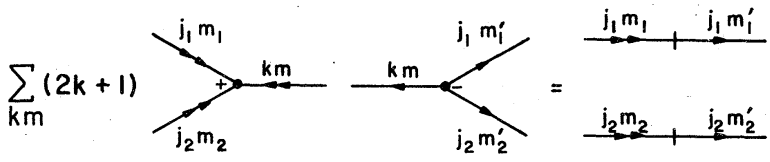

which represents the orthogonality relation

$$
\sum_{k m}(2 k+1)\left(\begin{array}{ccc}
m_{1} & m_{2} & m \\
j_{1} & j_{2} & k
\end{array}\right)\left(\begin{array}{lll}
j_{1} & j_{2} & k \\
m_{1}^{\prime} & m_{2}^{\prime} & m
\end{array}\right)=\delta_{m_{1} m_{1}^{\prime}} \delta_{m_{2} m_{2}^{\prime}} .
$$

Here we use the blocks $\alpha$ and $\beta$ to represent arbitrary diagrams either open or closed. By open or closed, we mean that the diagram either has or hasn't any free angular-momentum lines; for example,

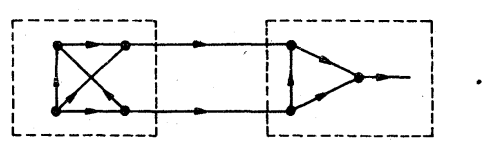

The diagram block on the left is closed while the one on the right open. Rule (2):
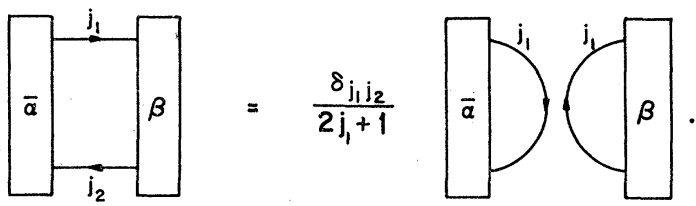

(A2)

Here $\bar{\alpha}$ denotes a closed diagram. Since derivation of this transformation rule is more involved, we refer the reader to Refs. 25 and 26 for its proof. Note that for a null block $\beta$, rule (A2) becomes

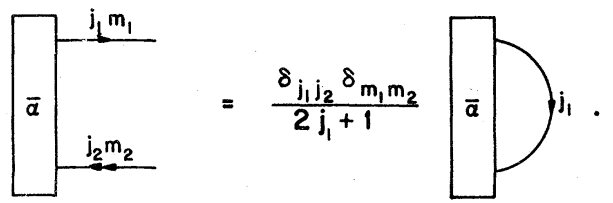

This transformation rule results from the rotational invariance of the diagram.

From these two fundamental rules, we can easily derive the following additional useful rules:
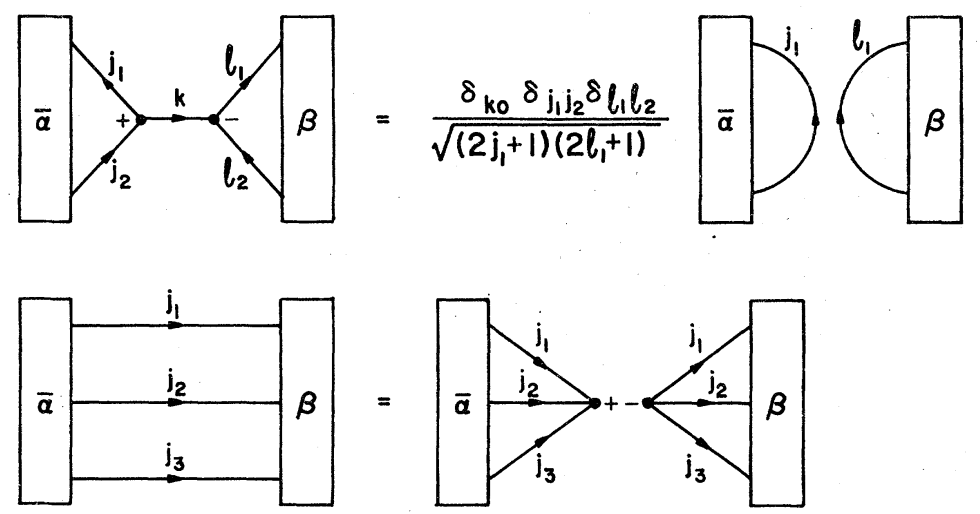


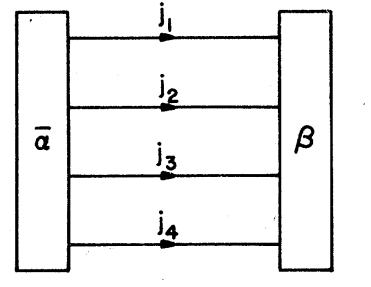

The transformation rules (A1) and (A2) and the rules derived from them allow one to rearrange diagrams or to factor out basic diagrams whose analytical values have been tabulated. Some of those basic diagrams are presented in Appendix B. For manipulating diagrams, note that the change in direction of a linked angular-momentum line $j$ will only introduce a phase factor $(-)^{2 j}$, and a sign change at the node $\left(j_{1}, j_{2}, j_{3}\right)$ will introduce a phase factor $(-)^{j_{1}+j_{2}+j_{3}}$. For more details of the transformation rules, see Refs. 25 and 26.

APPENDIX B: ANALYTICAL VALUES OF SOME BASIC DIAGRAMS

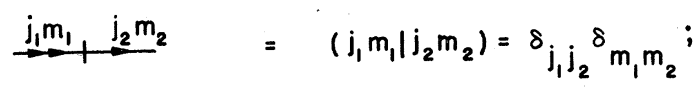<smiles>IC1CCCCCCC1</smiles>

$$
=2 j+1 ;
$$

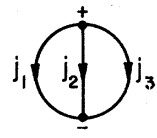
$=\left\{j_{1} j_{2} j_{3}\right\}$, triangular delta 34 $= \begin{cases}1 & \text { if }\left|j_{1}-j_{2}\right| \leqslant j_{3} \leqslant\left(j_{1}+j_{2}\right), \\ 0 & \text { otherwise; }\end{cases}$

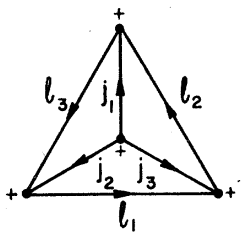

$=\left\{\begin{array}{lll}j_{1} & j_{2} & j_{3} \\ l_{1} & l_{2} & l_{3}\end{array}\right\}, 6-j$ symbol $;$

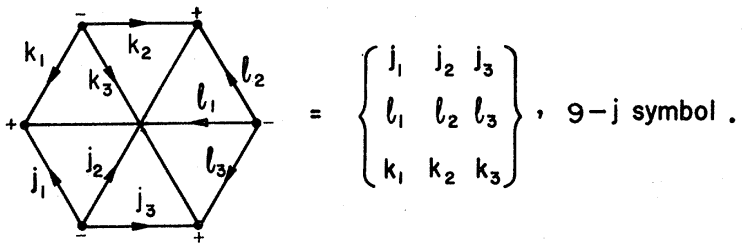

APPENDIX C: MATRIX ELEMENTS OF THE MUTUAL SPIN-ORBIT INTERACTION BETWEEN CONFIGURATIONS HAVING TWO ELECTRONS OUTSIDE CLOSED SHELLS

We summarize here the example, treated in Sec. IV, of the matrix element of mutual spin-orbit interaction between configurations having two electrons outside closed shells. In addition, we simplify our formulas for the special case of interaction between the two-electron configurations $n s n^{\prime} l^{\prime}$ and $n^{\prime \prime} s n^{\prime \prime \prime} l^{\prime \prime \prime}$ for a comparison with previously published results $\mathbf{s}^{13,21(\mathrm{a}), 21(\mathrm{~b})}$ and for application to $n s-$ subshell photoionization in the rare gases. ${ }^{35}$ As is known, ${ }^{21(b)}$ the results previously tabulated by Marvin $^{13}$ and by Jucys and Dagy ${ }^{21(a)}$ are in error due to their omission of integrals of the type $V^{\boldsymbol{l}}$. Furthermore, these previously tabulated results $^{13,21(a), 21(b)}$ are given, generally, for interactions within the same configuration, whereas our general formula treats the case of different interacting configurations. Note especially that even if the orbital angular-momentum quantum numbers of the interacting configurations are the same, the fact that the principal quantum numbers differ in the two configurations gives rise to additional radial integrals. For example, in the special case of interacting two-electron configurations $n_{a} l_{a} n_{b} l_{b}$ and $n_{c} l_{a} n_{d} l_{b}$ (where the orbital angularmomentum quantum numbers are the same) the interaction matrix element contains certain radial integrals which vanish when $n_{c}=n_{a}$ and $n_{d}=n_{b}$.

Matrix elements of the mutual spin-orbit interaction between configurations having two electrons outside closed shells can be summarized from Eqs. (42) and (57) for each of the following three cases:

(i) Nonequivalent outer-electron orbitals:

$$
\begin{aligned}
& \left\langle\phi_{a b}(12) \phi_{\text {closed }}\left|\sum_{i \neq j} V_{i j}\right| \phi_{c d}^{\prime}(12) \phi_{\text {closed }}\right\rangle_{A}=D(a b ; c d)+(-)^{l_{c}+\boldsymbol{l}_{d}+\boldsymbol{L}^{\prime}+s^{\prime}} D(a b ; d c) \\
& +(-)^{L+S+L^{\prime}+s^{\prime}} D(b a ; d c)+(-)^{l_{a}+l_{b}+L+s} D(b a ; c d) \\
& +\sum_{n_{i} \boldsymbol{t}_{i}}^{\text {closed }}\left\{(-)^{l_{a}+l_{b}+L+s} \delta_{a d}[D(b i a ; c i d)-E(b i a ; i c d)]\right. \\
& +(-)^{L+s+L^{\prime}+s^{\prime}} \delta_{a c}[D(b i a ; d i c)-E(b i a ; i d c)]+\delta_{b d}[D(a i b ; c i d)-E(a i b ; i c d)] \\
& \left.+(-)^{l_{c}+\boldsymbol{t}_{d}+L^{\prime}+\boldsymbol{s}^{\prime}} \delta_{b c}[D(a i b ; d i c)-E(a i b ; i d c)]\right\} \text {, }
\end{aligned}
$$


where $a, b, c, d$ in $\delta_{a d}$, etc. stand symbolically for all the quantum numbers of the respective orbitals.

(ii) Orbitals $a$ and $b$ are equivalent:

$$
\begin{aligned}
\left\langle\phi_{a a}(12) \phi_{\text {closed }}\left|\sum_{i \neq j} V_{i j}\right| \phi_{c d}^{\prime}(12) \phi_{\text {closed }}\right\rangle_{A}= & \sqrt{2}\left[D(a a ; c d)+(-)^{L^{\prime}+s^{\prime}} D(a a ; d c)\right] \\
& +\sqrt{2} \sum_{n_{i} l_{i}}^{\text {closed }}\left\{-\delta_{a d}[D(\text { aia } ; \text { cid })-E(\text { aia } ; \text { icd })]\right. \\
& \left.+(-)^{L^{\prime}+s^{\prime}} \delta_{a c}[D(\text { aia } ; \text { dic })-E(\text { aia } ; \text { idc })]\right\} .
\end{aligned}
$$

(iii) Orbitals $a$ and $b$ are equivalent, and orbitals $c$ and $d$ are equivalent:

$$
\left\langle\phi_{a a}(12) \phi_{\text {closed }}\left|\sum_{i \neq j} V_{i j}\right| \phi_{c c}^{\prime}(12) \phi_{\text {closed }}\right\rangle_{A}=2 D(a a ; c c)+2 \delta_{a c} \sum_{n_{i} \iota_{i}}^{\text {closed }}[D(a i a ; c i c)-E(a i a ; i c c)] \text {. }
$$

In Eqs. (C1)-(C3), $D(a b ; c d), D(a i b ; c i d), E(a i b ; i c d)$, and their permutations are defined by Eqs. (40), (49), and $(55)$, respectively.

For the special case of interacting two-electron configurations in which $l_{a}=l_{c}=0$, Eq. (C1) gives the matrix element between the triplet terms $\left({ }^{3} L_{J=L},{ }^{3} L_{J=L}\right)$ as

$$
-\left[3 /\left(2 l_{b}+1\right)\right]\left[\left(2 l_{b}+1\right) R_{0}^{(3)}(b a ; d c)+R_{l_{b}}^{(2)}(b a ; c d)+R_{l_{b}}^{(2)}(d c ; a b)\right]
$$

and between the singlet-triplet or triplet-singlet terms $\left({ }^{2 S+1} L_{J=L},{ }^{2 S^{\prime}+1} L_{J=L}\right)_{S \neq S^{\prime}}$ as

$$
\left\{\left[l_{b}\left(l_{b}+1\right)\right]^{1 / 2} /\left(2 l_{b}+1\right)\right\}\left[\left(2 l_{b}+1\right) R_{0}^{(3)}(b a ; d c)+(-)^{s} R_{l_{b}}^{(2)}(b a ; c d)+(-)^{s^{\prime}} R_{l_{b}}^{(2)}(d c ; a b)\right] .
$$

Or in terms of the following commonly used radial integrals

$$
\begin{aligned}
& N^{l}(a b ; c d)=\frac{\alpha^{2}}{4} \int_{0}^{\infty} r_{1}^{2} d r_{1} \int_{0}^{\infty} r_{2}^{2} d r_{2} R_{a}(1) R_{b}(2) \frac{r_{2}^{l}}{r_{1}^{l+3}} \theta\left(r_{1}-r_{2}\right) R_{c}(1) R_{d}(2), \\
& V^{l}(a b ; c d)=\frac{\alpha^{2}}{4} \int_{0}^{\infty} r_{1}^{2} d r_{1} \int_{0}^{\infty} r_{2}^{2} d r_{2} R_{a}(1) R_{b}(2) \frac{r_{<}^{l}}{r_{>}^{l+3}}\left(r_{2} \frac{\partial}{\partial r_{1}}-r_{1} \frac{\partial}{\partial r_{2}}\right) R_{c}(1) R_{d}(2),
\end{aligned}
$$

Eqs. (C4) and (C5) become

$$
\left[3 /\left(2 l_{b}+1\right)\right]\left[\left(2 l_{b}+1\right) N^{0}(b a ; d c)+\left(l_{b}+1\right) N^{l_{b}-2}(b a ; c d)-l_{b} N^{l} b(a b ; d c)+V^{l_{b}-1}(a b ; d c)\right]
$$
and

$$
\begin{aligned}
\left\{\left[l_{b}\left(l_{b}+1\right)\right]^{1 / 2} /\left(2 l_{b}+1\right)\right\}\{ & -\left(2 l_{b}+1\right) N^{0}(b a ; d c) \\
& \left.-(-)^{S^{\prime}}\left[\left(l_{b}+1\right) N^{l_{b}-2}(b a ; c d)-l_{b} N^{l} b(a b ; d c)+V^{l_{b}-1}(a b ; d c)+2 \boldsymbol{R}_{l_{b}}^{(2)}(b a ; c d)\right]\right\} .
\end{aligned}
$$

In particular cases, these formulas give the correct version of previously tabulated results. ${ }^{13,21(a)}$ In making comparisons with Refs. 13 and 21(a), note that we use $L S$ coupling whereas these other works use $S L$ coupling. Note also that (C5) and $\left(\mathrm{C5}^{\prime}\right)$ are generally not symmetrical with respect to interchange of $S$ and $S^{\prime}$.

*Supported in part by the U.S. Energy Research and Development Administration under Contract No. EY-76-S-02-2892

${ }^{\dagger}$ Work supported in part by the Alfred P. Sloan Foundation.

${ }^{1}$ E. U. Condon and Shortley, The Theory of Atomic Spectra (Cambridge University, Cambridge, 1957).

${ }^{2}$ J. C. Slater, Quantum Theory of Atomic Structure (McGraw-Hill, New York, 1960), Vols. I and II.

${ }^{3}$ W. Eissner, M. Jones, and H. Nussbaumer, Comput. Phys. Commun. 8, 270 (1974).

${ }^{4}$ A. W. Weiss, Bull. Am. Phys. Soc. 21, 1264 (1976).

${ }^{5}$ U. Fano, Comments At. Mol. Phys. 2, 30 (1970).

${ }^{6}$ P. A. M. Dirac, Proc. R. Soc. Lond. A 117, 610 (1928); P. A. M. Dirac, The Principles of Quantum Mechanics, 4th ed. (Oxford University, London, 1958).

${ }^{7}$ A. I. Akhiezer and V. B. Berestetskii, Quantum Electrodynamics (Wiley, New York, 1965).

${ }^{8}$ G. Breit, Phys. Rev. 34, 553 (1929).

${ }^{9}$ W. Pauli, Z. Phys. 43, 601 (1927).

${ }^{10} \mathrm{H}$. A. Bethe and E. E. Salpeter, Quantum Mechanics of One- and Two-Electron Atoms (Springer-Verlag, Berlin, 1957).

${ }^{11}$ E. David, Z. Phys. 91, 289 (1934).

${ }^{12}$ G. Araki, Proc. Phys. Math. Soc. Jpn. 21, 592 (1939).

${ }^{13}$ H. H. Marvin, Phys. Rev. 71, 102 (1947).

${ }^{14}$ R. H. Garstang, Mon. Not. R. Astron. Soc. 111, 115 (1951).

${ }^{15}$ J. P. Elliott, Proc. R. Soc. Lond. A 218, 345 (1953).

${ }^{16}$ H. Horie, Prog. Theor. Phys. 10, 296 (1953). 
${ }^{17}$ M. Blume and R. E. Watson, Proc. R. Soc. Lond. A $\underline{270}$, 127 (1962); 271, 565 (1963).

${ }^{18}$ M. Blume, A. J. Freeman, and R. E. Watson, Phys. Rev. A 134, 320 (1964).

${ }^{19}$ C. Fróese, Astrophys. J. 145, 934 (1966); Can. J. Phys. 45, 1501 (1967).

${ }^{20}$ D. R. Beck, J. Chem. Phys. 51, 2171 (1969).

${ }^{21}$ (a) A. P. Jucys and R. S. Dagys, Liet. TSR Mokslu Akad. Dar. Ser. B, 1 (21), 41 (1960); 59 (1960). (b) A. P. Jucys, R. S. Dagys, J, Vizbaraite, and S. Zvironaite, Liet. TSR Mokslu Akad. Dar. Ser. B, 3 (26), 53 (1961). (c) R. Karazija, J. Vizbaraite, and A. P. Jucys, Litov. Fiz. Sb. 6, 487 (1966); J. Vizbaraite, R. Karazija, J. Grudzinskäs, and A. P. Jucys, Litov. Fiz. Sb. 7, 25 (1967).

${ }^{22}$ A. P. Jucys and A. J. Savukynas, Mathematical Foundations of the Atomic Theory (Mintis, Vilnius, 1973) (in Russian).

${ }^{23}$ M. Jones, J. Phys. B $\underline{3}, 1571$ (1970); 4, 1422 (1971).

${ }^{24}$ W. Eissner and H. Nussbaumer, J. Phys. B 2,1028 (1969).

${ }^{25}$ A. P. Jucys, I. B. Levinson, and V. V. Vanagas, The
Theory of Angular Momentum (Isr. Prog. for Sci. Trans., Jerusalem, 1962).

${ }^{26} \mathrm{E}$. El Baz and B. Castel, Graphical Methods of Spin Algebras in Atomic, Nuclear, and Particle Physics (Marcel Dekker, New York, 1972).

${ }^{27}$ J. S. Briggs, Rev. Mod. Phys. 43, 189 (1971).

${ }^{28}$ E. P. Wigner, Group Theory (Academic, New York, 1959).

${ }^{29} \mathrm{~A}$. de-Shalit and I. Talmi, Nuclear Shell Theory (Academic, New York, 1963).

${ }^{30}$ M: E. Rose, Elementary Theory of Angular Momentum (Wiley, New York, 1957).

${ }^{31}$ A. R. Edmonds, Angular Momentum in Quantum Mechanics (Princeton University, Princeton, 1957).

${ }^{32}$ W. D. Klotz, Comput. Phys. Commun. $\underline{9}, 102$ (1975).

${ }^{33}$ L. H. Thomas, Nature 117, 514 (1926).

${ }^{34}$ Some authors call the triangular delta "the $3-j$ symbol", and call Wigner's $3-j$ symbol "the 3-jm symbol", which reflects the $m$ dependence of Wigner's $3-j$ symbol. However, we shall adopt the common usage and reserve "the 3- $j$ symbol" for Wigner's $3-j$ symbol.

${ }^{35}$ K. -N. Huang and A. F. Starace (unpublished). 